\title{
Intra-Voxel Incoherent Motion at 7 Tesla to quantify human spinal cord perfusion: limitations and promises
}

\author{
Simon Lévy ${ }^{1-4}$, Stanislas Rapacchi ${ }^{1,2}$, Aurélien Massire ${ }^{1,2,4}$, Thomas Troalen ${ }^{5}$, Thorsten \\ Feiweier $^{6}$, Maxime Guye ${ }^{1,2}$, Virginie Callot ${ }^{1,2,4}$
}

\author{
Affiliations \\ ${ }^{1}$ Aix-Marseille Univ, CNRS, CRMBM, Marseille, France \\ ${ }^{2}$ APHM, Hopital Universitaire Timone, CEMEREM, Marseille, France \\ ${ }^{3}$ Aix-Marseille Univ, IFSTTAR, LBA, Marseille, France \\ ${ }^{4}$ iLab-Spine International Associated Laboratory, Marseille-Montreal, France-Canada \\ ${ }^{5}$ Siemens Healthcare SAS, Saint-Denis, France \\ ${ }^{6}$ Siemens Healthcare GmbH, Erlangen, Germany
}

\section{Corresponding author}

Virginie Callot

CRMBM-CEMEREM, Aix-Marseille Université, Hôpital Universitaire Timone

27 bd Jean Moulin

13385 Marseille cedex 05, France

Office: +33491388465 | Email: virginie.callot@univ-amu.fr 


\section{Abstract}

Purpose: To develop a noninvasive technique to map human spinal cord (SC) perfusion in-vivo. More specifically, to implement an Intra-Voxel Incoherent Motion (IVIM) protocol at ultrahigh field for the human SC and assess parameters estimation errors.

Methods: Monte-Carlo simulations were conducted to assess estimation errors of two standard IVIM fitting approaches (two-step versus one-step fit) over the range of IVIM values reported for the human brain and for typical SC diffusivities. Required Signal-toNoise Ratio (SNR) was inferred for estimation of the parameters product $f_{I V I M} D^{*}$ (microvascular fraction times pseudo-diffusion coefficient) within 10\% error margins. Invivo IVIM imaging of the SC was performed at $7 \mathrm{~T}$ in 6 volunteers. An image processing pipeline is proposed to generate IVIM maps and register them for an atlas-based regionwise analysis.

Results: Required $\mathrm{b}=0$ SNRs for $10 \%$ error estimation on $f_{I V I M} D^{*}$ with the one-step fit were 159 and 185 for diffusion-encoding perpendicular and parallel to the SC axis, respectively. Average in-vivo $\mathrm{b}=0 \mathrm{SNR}$ within cord was $141 \pm 79$, corresponding to estimation errors of $12.7 \%$ and $14.7 \%$ according to numerical simulations. Slice- and group-averaging reduced noise in IVIM maps, highlighting the difference in perfusion between gray and white matter. Mean \pm standard deviation $f_{I V I M}$ and $D^{*}$ values across subjects within gray (respectively white) matter were $16.0 \pm 1.7(15.0 \pm 1.6) \%$ and $11.4 \pm 2.9(11.5 \pm 2.4) \times 10^{-3} \mathrm{~mm}^{2} / \mathrm{s}$.

Conclusion: Single-subject data SNR at 7T was insufficient for reliable perfusion estimation. However, atlas-averaged IVIM maps highlighted the higher microvascular fraction of gray matter compared to white matter, providing first results of healthy human SC perfusion mapping with MRI.

Keywords: spinal cord, 7T MRI, perfusion, ultrahigh field MRI, IVIM, intravoxel incoherent motion 


\section{Introduction}

There is a crucial need for noninvasive assessment of spinal cord (SC) perfusion in diagnosing and stratifying the severity of chronic pathology. In most SC compressive injuries (e.g., trauma, degenerative cervical myelopathy), perfusion impairment is the precursor of tissue degeneration leading to axonal loss or demyelination followed by clinical symptoms (e.g., pain, paralysis) ${ }^{1}$. As SC decompression implies major and invasive surgery, the surgical benefit has to be accurately evaluated. So far, clinicians' decision relies on clinical presentation and MRI findings such as CSF effacement, cord deformation and $\mathrm{T}_{1} / \mathrm{T}_{2}$ hypo/hyperintensity ${ }^{2}$; however, these markers are indirect, not specific and often limited to establishing irreversible tissue damage ${ }^{3}$. In line with ongoing studies evaluating the diagnosis and prognosis performances of quantitative MRI metrics ${ }^{4,5}$, monitoring in-vivo SC perfusion would provide an earlier biomarker of tissue viability and consequently help clinicians in therapeutic orientation and prognosis.

Perfusion imaging techniques successfully applied to the brain face multiple challenges when translated to SC. First of all, SC is a very small structure $\left(\sim 8 \mathrm{x} 13 \mathrm{~mm}^{2}\right.$ ellipse in transverse section ${ }^{6}$ ) that requires high spatial resolution. Moreover, multiple physiological sources of signal dropout may hamper the acquisition: CSF pulsation with heartbeat as well as respiration lead to complex movements of the cord, both in inferiorsuperior direction and in transverse plane ${ }^{7}$. The longitudinal and tubular shape together with vertebrae protecting the cord, and the proximity of the lungs are additional challenges for both static $\left(\mathrm{B}_{0}\right)$ and $\mathrm{RF}\left(\mathrm{B}_{1}{ }^{+}\right)$fields homogeneity. Last but not least, perfusion within $\mathrm{SC}$, driven by capillaries (diameter $<10 \mu \mathrm{m}$ ), is expected to be similar to brain perfusion (according to results in mice $^{8}$ ), i.e. very low compared to other organs such as kidneys ${ }^{9}$ or liver ${ }^{10}$. High sensitivity of the technique is thus needed.

Global SC perfusion has been probed using contrast agent-based techniques. Dynamic Susceptibility Contrast (DSC) imaging was performed in patients with cervical stenosis to relate average SC perfusion measurements within a global region to neurological scores and compression degree ${ }^{11-13}$ but neither mapping of SC perfusion nor distinction between SC regions were performed. Technical feasibility of DCE imaging for perfusion of intradural spinal lesions at cervical level was also assessed at $1.5 \mathrm{~T}$ and $3 \mathrm{~T}^{14}$ but again quantification was performed globally for the whole lesion region, which involved high perfusion levels with respect to healthy tissue. 
Given the increasing concerns regarding gadolinium deposition in bone and brain even in patients with normal renal function and intact blood-brain barriers ${ }^{15,16}$, motivation for endogenous contrast mechanisms has risen. Moreover, with endogenous methods, acquisition duration can be traded for SNR, which cannot be done with exogenous techniques where acquisition time and SNR are inherently limited by contrast agent first pass duration and relaxivity. Leveraging SNR is crucial in SC imaging to achieve sufficient resolution.

Arterial Spin Labeling (ASL) is a common endogenous method which has been extensively applied in human brain investigations ${ }^{17}$. The technique also demonstrated potential in the mouse SC where preclinical scanners enable global tagging strategies ${ }^{8,18}$. Yet, this technique is hardly applicable to human as such tagging strategies are limited by hardware capabilities given the human body size. Furthermore, unlike in the brain, the multiple sources of perfusion of the SC tissue and the complexity and interindividual variability of the vascular network render local tagging strategies nontrivial. Indeed, two groups attempted to map SC perfusion at $1.5^{19}$ and $3 \mathrm{~T}^{20}$ with such techniques but experienced poor reliability and reproducibility in their results and no further study was published since then.

7T MRI appears as a promising clinical avenue to increase SNR and improve sensitivity. However, ASL remains problematic at this field strength since labelling pulses require high energy and thus may encounter Specific Absorption Rate (SAR) limits and suffer from $B_{1}$ inhomogeneity issues. Indeed, the limited efficacy and transmit field homogeneity of the currently available $\mathrm{Tx} / \mathrm{Rx}$ coils for cervical spine imaging at $7 \mathrm{~T}$ jeopardizes the efficient initiation of a labelling plane for continuous and pseudocontinuous ASL. Parallel transmission and dielectric pads can alleviate this constraint ${ }^{21}$ but remain emerging technologies.

Given the major progress in SC diffusion MRI at 7T 22,23 , Intra-Voxel Incoherent Motion (IVIM) imaging emerges as a promising technique for SC perfusion imaging. This technique aims at quantifying the signal decrease at low b-values induced by blood water circulation through capillaries mimicking a Brownian motion random-walk at larger scale ${ }^{24}$. Sensitivity to perfusion is therefore achieved through diffusion gradients, which does not bring additional challenges at ultrahigh field, unlike ASL relying on the efficacy of the inversion pulse. It also does not rely on in-flow blood labelling or Arterial Input Function to the tissue, which is an asset given the multiple sources of perfusion of the SC. Furthermore, IVIM has already been extensively applied in humans to several 
organs (e.g., brain ${ }^{25-30}$, kidneys ${ }^{31}$, liver ${ }^{10,32,33}$, heart ${ }^{34}$ or pancreas ${ }^{35-37}$ ), but remains unexplored in SC.

In this study, we present a comprehensive protocol for IVIM mapping of the human SC, exploiting increased SNR from ultrahigh field strength. An optimization of data acquisition, processing and parameter fitting is proposed, carefully considering estimation errors on derived IVIM parameters. This work finally showcases, to the best of our knowledge, the first perfusion-related in-vivo maps of the human SC and quantifies the IVIM parameters within SC regions. 


\section{Methods}

\subsection{Simulations}

Two standard fitting approaches to estimate IVIM parameters were considered: the "two-step segmented"33,38 approach and the "one-step"38 approach. Their implementations are sketched in Figure 1. Both fitting pipelines were implemented in Python (2.7), building upon the LMFIT module (lmfit.github.io/lmfit-py), which facilitates distribution and validation of implementation as it is all open-source.

Software and implementations can have a significant impact on accuracy and precision of parameter estimations ${ }^{25,28,39}$ regardless of computational speed. Therefore, inspired by the work of Pekar et al. ${ }^{38}$, we performed Monte-Carlo simulations to assess their performance under different physiological and SNR conditions. Ranges of possible values for IVIM parameters were defined according to ranges of values found in brain literature ${ }^{25-28,39-42}$ and typical SC radial and axial diffusivities ${ }^{23,43}$ :

- Visible microvascular volume fraction $f_{I V I M}(\%): 1$ to 30

- Pseudo-diffusion coefficient $D^{*}\left(\mathrm{~mm}^{2} / \mathrm{s}\right): 3.0 \times 10^{-3}$ to $35.0 \times 10^{-3}$

- Pure diffusion coefficient $D\left(\mathrm{~mm}^{2} / \mathrm{s}\right): 0.3 \times 10^{-3}\left(D_{\perp}\right.$, diffusivity in the $\mathrm{SC}$ transverse plane) and $1.5 \times 10^{-3}\left(D_{\|}\right.$, diffusivity along the $\mathrm{SC}$ axis)

The IVIM representation of the signal ${ }^{24}$ is given by (Eq. 1):

$$
S=S_{b=0} e^{-b \cdot D}\left(f_{I V I M} e^{-b \cdot D^{*}}+1-f_{I V I M}\right)
$$

To assess performance of fitting algorithm, synthetic data were generated using (Eq. 1) and ranging a large number of b-values inspired by IVIM protocols in brain $25,26,40,44$ and Diffusion Tensor Imaging practice in $\mathrm{SC}^{45,46}$ : 5, 10, 15, 20, 30, 50, 75, 100, 125, 150, $200,250,600,700,800 \mathrm{~s} / \mathrm{mm}^{2}$. Given that the in-vivo fitted data result from the averaging of multiple repetitions of magnitude images, according to the Central Limit Theorem, the noise distribution in such data can be assumed Gaussian. Therefore, random Gaussian noise was added to synthetic data with SD matching realistic SNR as described below. Finally, mean absolute estimation error across $\mathrm{N}=1000$ random noise draws was calculated for each fitted parameter according to:

$$
\operatorname{error}(\%)=\frac{100}{N} \times \sum_{i=1}^{N} \frac{\mid \text { estimated } \text { value }_{i}-\text { true value } \mid}{\text { true value }}
$$

Three types of simulations were performed: 


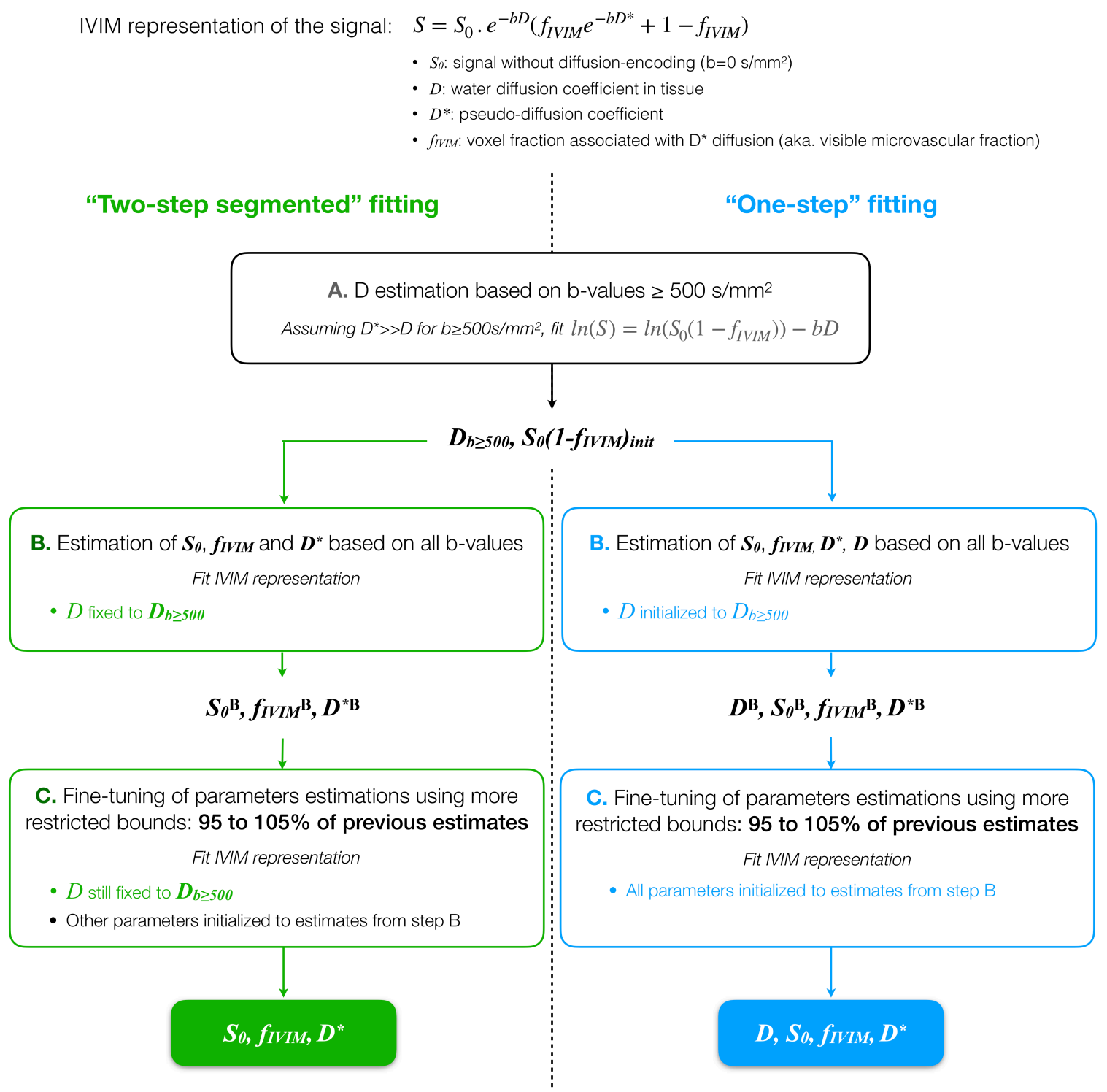

Figure 1. Implementation of the two "standard" fitting approaches encountered in literature: "two-step segmented" (left side) and "one-step" (right side) fitting. Each frame represents a fitting process and includes fit details (e.g. parameters initialization) while outbound arrows point to parameter estimates resulting from this fitting process. Step A employed the Conjugate Gradient optimization method due to its computational speed while steps B and C employed the Differential Evolution method to escape local optima. For both approaches, a first estimation of $D$ is performed based on high b-values signal (step A). This first estimate is definitive for the "two-step segmented" approach but is only used as initial value for the "one-step" approach along with intercept $\left(S_{0}(1-\right.$ $\left.f_{I V I M}\right)_{\text {init }}$ ). After step B, all parameters are fitted; step C consists in a fine-tuning of the fit, constraining parameter estimation to $[95 \% ; 105 \%]$ of the value obtained at previous step. Parameter constraints at step B were defined based on extreme values found in published IVIM studies within the brain white and gray matter ${ }^{25-28,39-42}$ and typical SC radial and axial diffusivities ${ }^{23,43}$. 
1) Estimation errors were assessed with infinite $\operatorname{SNR}\left(S D_{\text {noise }}=0\right)$ for both fitting approaches and for all parameter values within the defined ranges. These simulations served to verify whether algorithms could retrieve true parameter values on perfect data given the b-value distribution used.

2) Estimations errors were assessed for realistic SNR values of 60, 120 and 180. These values corresponded to the minimum, mean and maximum across subjects of the average voxel-wise SNR within SC measured in-vivo at $\mathrm{b} \approx 0$.

3) The minimum required SNR for $b=0$ data to get an error $\leq 10 \%$ on the parameter $f_{I V I M} D^{*}$ (product of $f_{I V I M}$ and $D^{*}$, related to blood flow $^{47}$ ) was computed for all parameter values within the defined ranges and for the one-step approach.

To get the corresponding required SNR for higher b-values (e.g. $b=800 \mathrm{~s} / \mathrm{mm}^{2}$ with $D=1.5 \times 10^{-3} \mathrm{~mm}^{2} / \mathrm{s}$ in the $\mathrm{SC}$ for diffusion weighting along inferior-superior direction), one just needs to multiply it by $e^{-b D}$ (i.e. here 0.3 ), assuming that the microvascular compartment contribution to the signal at such high b-values can be neglected $\left(D^{*}>>D\right)$. SNR of diffusion-weighted images depends on the orientation of the diffusion gradient (as accounted for in the diffusion coefficient $D$ ).

Fitting algorithms (Figure 1) were optimized through simulations 1) and 2), especially regarding the fit optimization method (e.g., Trust-Region Reflective least squares, Levenberg-Marquardt, Brute Force). The Differential Evolution method ${ }^{48}$, as implemented in LMFIT module was preferred to avoid local optima solutions and to provide speed and precision in parameters estimation.

\subsection{Data acquisition}

\subsubsection{Population and MR setup}

The study was approved by the local ethics committee and written consents were obtained from 8 healthy volunteers prior to MR examinations. Two subjects ( 1 male, 1 female) were dedicated to optimization of acquisition parameters and six (5 males, 1 female, mean age $\pm \mathrm{SD}=25.0 \pm 2.6$ years old) were scanned with the optimized protocol. Acquisitions were performed on a $7 \mathrm{~T}$ whole-body research system (Siemens Healthcare, Erlangen, Germany) with a commercial 8-channel cervical-spine transceiver surface coil (Rapid Biomedical GmbH, Rimpar, Germany) employed with the 8 Tx-channels 
hardware-combined into a single transmit system. The complete protocol (see more details in Supporting Information Table S1) included:

- Patient-specific tuning: localizer for slice positioning, coil voltage calibration, local $\mathrm{B}_{0}$ shimming within a $\sim 4 \times 3 \times 5 \mathrm{~cm}$ (Right-Left $\times$ Anterior-Posterior $\times$ InferiorSuperior) volume around the cord, $\mathrm{B}_{1}{ }^{+}$and $\mathrm{B}_{0}$ maps to inspect fields inhomogeneities and shimming performance

- Sagittal 2D turbo-spin echo imaging with $0.6 \times 0.6 \mathrm{~mm}^{2}$ in-plane resolution and $2.2 \mathrm{~mm}$ slice thickness for vertebral levels localization

- Pulse-triggered IVIM protocol (further described below)

- Axial 2D high-resolution $\left(0.4 \times 0.4 \mathrm{~mm}^{2}\right.$ in-plane, $5 \mathrm{~mm}$ slice thickness $)$ multi-echo gradient-echo (MGE) image for gray matter (GM) segmentation

Depending on the subject's heartbeat, total protocol time was $1 \mathrm{~h} 10-15 \mathrm{~min}$.

\subsubsection{IVIM acquisition protocol}

IVIM acquisitions were based on a prototype 2D single-shot diffusion-prepared spinecho EPI sequence. Six slices (5-mm thick with 1-mm gap) were centered at C3-C4 intervertebral disk (area minimizing partial volume effects induced by cord curvature in this cohort). C3-C4 coverage was guaranteed for all subjects. Phase was encoded along the R-L axis for robust parallel imaging given the coil configuration (see coil diagram in Massire et al. ${ }^{22}$ ).

As a low spinal cord perfusion level is expected according to brain studies ${ }^{25,49,50}$, sequence parameters were experimentally optimized to maximize SNR (Supporting Information Figure S1-A). Following acquisition parameters were investigated: diffusion scheme, minimal bandwidth, GRAPPA factor, partial Fourier, outer volume suppression with reduced FOV strategy, number of lines for GRAPPA calibration scans and partial Fourier reconstruction algorithm. Regarding diffusion scheme, as SNR decreases with longer TE, only short TE schemes were considered: the standard Stejskal-Tanner scheme (monopolar) and a modified version of it ("monopolar+"), applying diffusion gradients during the entire time between excitation and refocusing pulses, leaving no deadtime ${ }^{51}$. With the optimized parameter set, a minimum TE of $51.6 \mathrm{~ms}$ (effective $\delta / \Delta=12.2 / 13.3 \mathrm{~ms}$ ) and an SNR gain of $27 \%$ compared to the "base" protocol were obtained (see Supporting Information Figure S1-A and Table S1).

To mitigate effects of SC motion and CSF pulsation, acquisitions were synchronized on cardiac beat monitored with a pulse oximeter (no trigger delay). The number of cycles 
to acquire all slices $N_{R R}$ (determining the effective TR for each slice) was adapted to each subject's heartbeat duration $T_{R R}$ so as to get the highest SNR efficiency (SNR/time unit), which was calculated using the spin-echo signal expression derived from Bloch equations for a single refocusing pulse ${ }^{52}$ and $\mathrm{SC} \mathrm{T}_{1}$ values from the literature ${ }^{22}$ (Supporting Information Figure S1-B). The resulting effective TR per slice was $2.6 \mathrm{~s}$ in average. Considering that the maximum variation of $T_{R R}$ in healthy subjects would be about $0.2 \mathrm{~s}$, with a $T_{1}$ in the $\mathrm{SC}$ around $1.25 \mathrm{~s}^{22}$, the variations in longitudinal relaxation would be, in the worst case scenario (effective TR variation of $0.6 \mathrm{~s}$ with $N_{R R}=3$ ), about $6 \%$ at $\max$ $\left(1-e^{-T R_{e f f}} / T_{1}\right)$. Moreover, these potential variations would be eventually mitigated by the averaging over multiple repetitions in final data. Consequently, signal relaxation variations due to effective TR variations along acquisition were considered negligible in this study.

Finally, b-value distribution was empirically defined, inspired by IVIM protocols in brain $25,26,40,44$ and Diffusion Tensor Imaging practice in $\mathrm{SC}^{45,53}$, so as to focus sampling on low b-values $\left(\leq 250 \mathrm{~s} / \mathrm{mm}^{2}\right)$ to better probe the fast decaying signal related to perfusion. The number of b-values $N_{b}$ was also empirically defined in direct relation with the number of repetitions $N_{\text {rep }}$ per b-value, limiting the scan time to a maximum of $1 \mathrm{~h}$ for the IVIM protocol, which included 3 orthogonal diffusion-encoding directions (right-left, R-L, anterior-posterior, A-P, inferior-superior, I-S), i.e.:

$$
N_{b} \cdot N_{\text {rep }} \cdot N_{R R} \cdot T_{R R} \cdot 3<1 \text { hour }
$$

B-values were corrected to include imaging gradient contributions. Eventually, actual bvalue distribution was $5,10,15,20,30,50,75,100,125,150,200,250,600,700,800$ $\mathrm{s} / \mathrm{mm}^{2}$ with 30 repetitions per b-value, allowing slight variations between subjects and directions as explained above. Furthermore, to correct for EPI readout-related distortions, acquisitions were split into two distinct imaging sets of equal lengths $\left(N_{\text {rep }} / 2\right)$, in forward (right $>$ left) and reverse (left>right) phase-encoding direction for each diffusion-encoding direction.

\subsection{Data post-processing}

First, diffusion-weighted data were denoised using the local PCA algorithm developed by Manjón et al. ${ }^{54}$ across repetitions, for each b-value and independently for each phase-encoding direction. Second, the sub-pixel shifting method from Kellner et al. ${ }^{55}$ was applied to remove any potential Gibbs-ringing artifact. Third, a dedicated 
program (sct_dmri_moco) available within the Spinal Cord Toolbox ${ }^{56}$ (v3.1.1) was used for correction of cord motion throughout the acquisition. Fourth, EPI readout-related distortions were corrected using the topup program from $\mathrm{FSL}^{57}$ based on forward and reverse phase-encoded $b=0$ images and applying the least-square restoration method. Fifth and final step, all repetitions of equal b-value were averaged, ending up with one volume (6 slices) per b-value and diffusion-encoding direction.

\subsection{In-vivo SNR and data fitting}

As defined by Reeder et al. ${ }^{58}$, in-vivo SNR was calculated voxel-wise as the ratio of the mean signal across repetitions to the SD across repetitions. Calculation was performed on the lowest b-value images obtained, after the post-processing pipeline was applied. A factor of $\sqrt{N_{\text {rep }}}$ was applied to reflect the SNR value of the average across all repetitions, which is used as input data to the fitting algorithm. Voxel-wise SNR was averaged per subject across the entire SC and the minimum, maximum and mean values across subjects served as references for the simulations (section 2.1).

Based on simulation results (section 3.1), in-vivo data were fitted voxel-wise using the one-step approach described in Figure 1. Five IVIM parameter 3D maps $\left(f_{I V I M}, D^{*}, D, S_{0}\right.$ and $\left.f_{I V I M} D^{*}\right)$ were produced for each subject and each diffusion-encoding direction.

\subsection{Quantification}

For group analysis and quantification within regions of interest (ROIs), IVIM maps were registered to the PAM50 template ${ }^{59}$ and its white matter (WM) atlas ${ }^{60}$ using dedicated tools from the Spinal Cord Toolbox ${ }^{56,61,62}$ (v3.1.1) as described in Figure 2. 


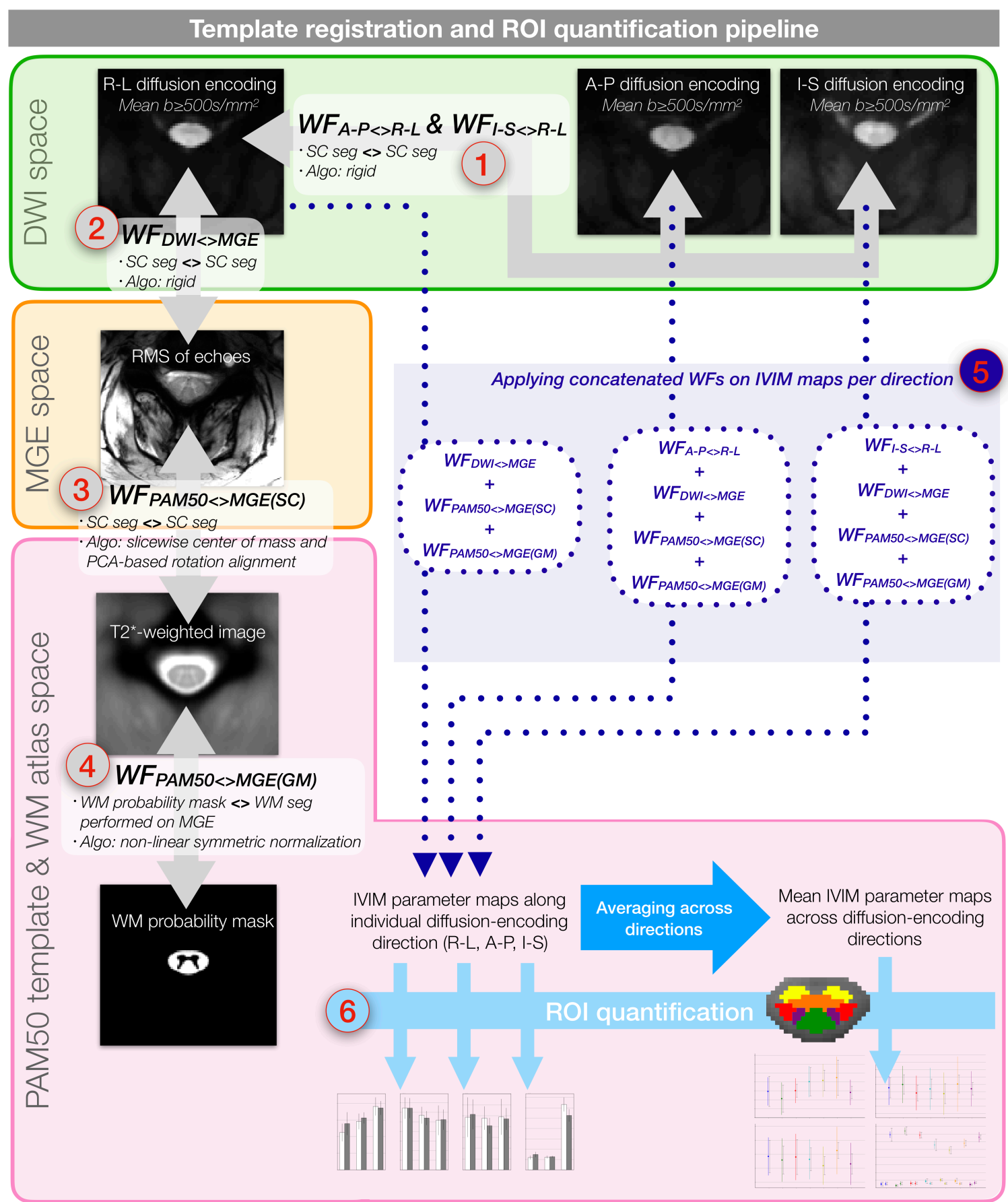

Figure 2. Template registration pipeline performed for every subject. (1) Data acquired with A-P and I-S diffusion encoding are registered (rigid transformation) to the data with $\mathrm{R}$-L diffusion encoding, based on the mean image across b-values $\geqslant 500 \mathrm{~s} / \mathrm{mm}^{2}$. (2) The $\mathrm{R}-\mathrm{L}$ diffusion-encoded mean image is registered (rigid transformation) to the RMS of all echoes of the high-resolution MGE image, yielding the Warping Field (WF) $W F_{D W I<>M G E}$. (3) The template is registered to MGE space based on SC segmentations ( $\mathrm{SC}$ seg) obtained with sct propseg ${ }^{61}$ and manual corrections when needed - yielding

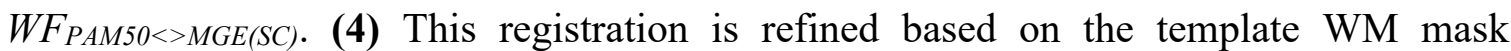
registered to MGE space and the WM segmentation (WM seg) performed on MGE image 


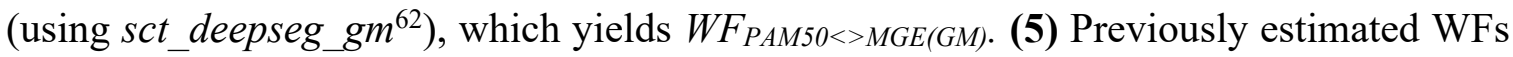
are concatenated to get final transformations between Diffusion-Weighted Images (DWI) and template spaces; those transformations are applied to every IVIM parameter maps for each diffusion-encoding direction. (6) After group-averaging, IVIM parameters are quantified within ROIs independently per diffusion-encoding direction as well as on the mean maps across the three directions (except for the diffusion coefficients $D_{R-L}, D_{A-P}, D_{I-}$ s). Note that the masks of ROIs were eroded at the interface with CSF in order to exclude voxels corrupted by elevated apparent CSF pseudo-diffusion and partial volume effects in IVIM maps (see Figure 7). Algo: registration algorithm. 


\section{Results}

\subsection{The one-step fitting approach performs better for the range of IVIM values expected in the human spinal cord}

For IVIM parameter values expected in the human SC, the one-step fitting approach showed better estimation performance than the two-step segmented approach, both on perfect $(\mathrm{SNR}=\infty)$ and noisy data (SNR levels measured in-vivo). This comparison can be found in Supporting Information Figure S2. The higher performance of the one-step approach is observed on all parameters, especially on $D$, which is due to the low sampling of high b-values (only $3 \mathrm{~b}$-values $\geq 600 \mathrm{~s} / \mathrm{mm}^{2}$ ); the inaccuracy in $D$ then propagates more importantly on the other parameters with the two-step segmented approach. Consequently, given the b-value distribution used, the one-step fitting approach is more appropriate for IVIM parameters fitting in the human SC.

More in details for the one-step fitting approach (Figure 3), the algorithm perfectly retrieves the true values of parameters with infinite SNR and estimation errors increase when SNR decreases. The parameter estimated with highest precision is $D$, then $f_{I V I M}$ and then $f_{I V I M} D^{*}$, and the parameter estimated with the largest error is $D^{*}$, as commonly reported in the IVIM literature. Interestingly, for high $D$ values $\left(D_{\|}\right)$, the error on $D$ is lower than for low $D$ values $\left(D_{\perp}\right)$ whereas it is higher for $f_{I V I M}, D^{*}$ and $f_{I V I M} D^{*}$. Indeed, signal decay induced by high $D$ is sharper at high b-values but then, the effect of the other IVIM parameters on the signal is more challenging to extract. Furthermore, errors increase when perfusion levels $\left(f_{I V I M}, D^{*}, f_{I V I M} D^{*}\right)$ decrease, as signal decay gets slower and smaller. For instance, with high SNR (180) and low $D\left(0.3 \times 10^{-3} \mathrm{~mm}^{2} / \mathrm{s}\right)$, estimation errors on $f_{I V I M}, D^{*}$ and $f_{I V I M} D^{*}$ exceed $100 \%$ (squares above orange levels) when $\left(f_{I V I M}\right.$, $\left.D^{*}\right)$ is lower than $\left(4.2 \%, 6.6 \times 10^{-3} \mathrm{~mm}^{2} / \mathrm{s}\right),\left(13.9 \%, 13.7 \times 10^{-3} \mathrm{~mm}^{2} / \mathrm{s}\right)$ and $\left(1 \%, 6.6 \times 10^{-3}\right.$ $\mathrm{mm}^{2} / \mathrm{s}$ ), respectively. Finally, when $f_{I V I M}$ is around $16 \%$ and $D^{*}$ around $11.5 \times 10^{-3} \mathrm{~mm}^{2} / \mathrm{s}$ (as measured in-vivo, see below) and potentially lower $(\sim 10 \%)$, the expected error on $f_{I V I M}, D^{*}$ and $f_{I V I M} D^{*}$ is around 15,30 and $10 \%$ with $\mathrm{SNR}=180$, respectively.

\subsection{High $\mathrm{SNR}$ is required for accurate estimation of $f_{I V I M} D^{*}$}

In the range of considered IVIM values, high $b=0 \mathrm{SNR}(\geq 70)$ is required to estimate $f_{I V I M} D^{*}$ within $10 \%$ error margins (Figure 4 ). SNR higher than 400 is needed if $f_{I V I M} \leq$ $7.4 \%$ and/or $D^{*} \leq 3.0 \times 10^{-3} \mathrm{~mm}^{2} / \mathrm{s}$. 


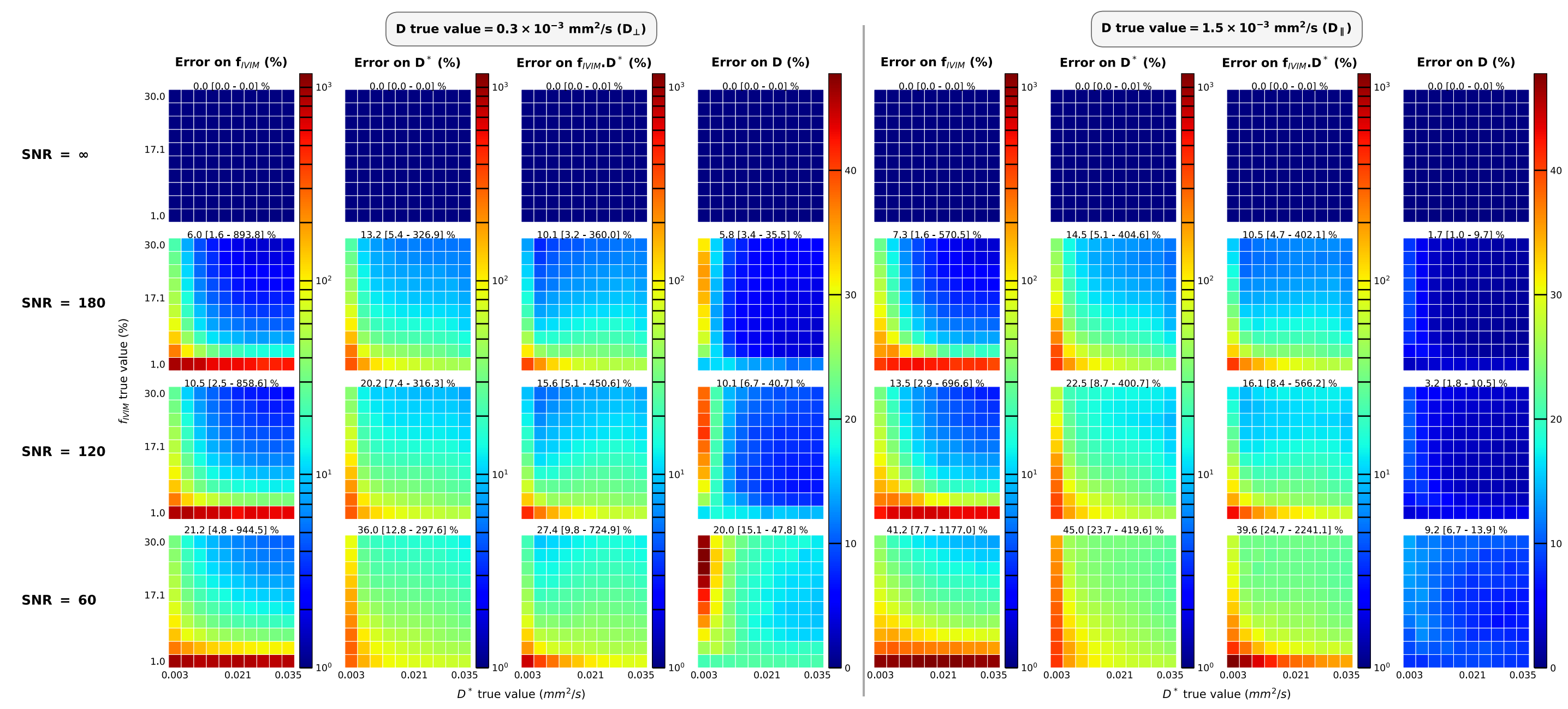

Figure 3. Estimation errors on each IVIM parameter according to $\left(f_{I V I M}, D^{*}, D\right)$ true values for the one-step fitting approach with different SNR levels: $\infty$ (no noise), 180 (maximum in-vivo SNR), 120 (mean), 60 (minimum). On the $y$-axis of each graph, $f_{I V I M}$ varies from 1 to $30 \%$ while on the $x$-axis $D^{*}$ varies from 3 to $35 \times 10^{-3} \mathrm{~mm}^{2} / \mathrm{s}$ (bounds defined according to IVIM literature in brain white and gray matter as described in section 2.1); on the left side, $D=0.3 \times 10^{-3} \mathrm{~mm}^{2} / \mathrm{s}$, similar to diffusivity in SC transverse plane $\left(D_{\perp}\right)$, while on the right side $D=1.5 \times 10^{-3} \mathrm{~mm}^{2} / \mathrm{s}$, similar to diffusivity along SC axis $\left(D_{\|}\right)$. Median [min-max] errors are indicated on top of each graph. 
Due to crosstalk between $f_{I V I M}$ and $D^{*}$ (shared sensitivity to the same physiological process), their individual error can exceed $10 \%$ while their product $f_{I V I M} D^{*}$ offers an accuracy below $10 \%$ (e.g. for $f_{I V I M}, D^{*}=13.9 \%, 10.1 \times 10^{-3} \mathrm{~mm}^{2} / \mathrm{s}$ ).

Of note, increased $D$ requires higher SNR for the same accuracy, which stands as a challenge for IVIM along the SC axis. Figure 4 also shows that $f_{I V I M}$ is preponderant over $D^{*}$ in determining the required SNR.

Minimum required SNR to get an error $\leq 10 \%$ on $f_{/ V I M} \cdot D^{*}$
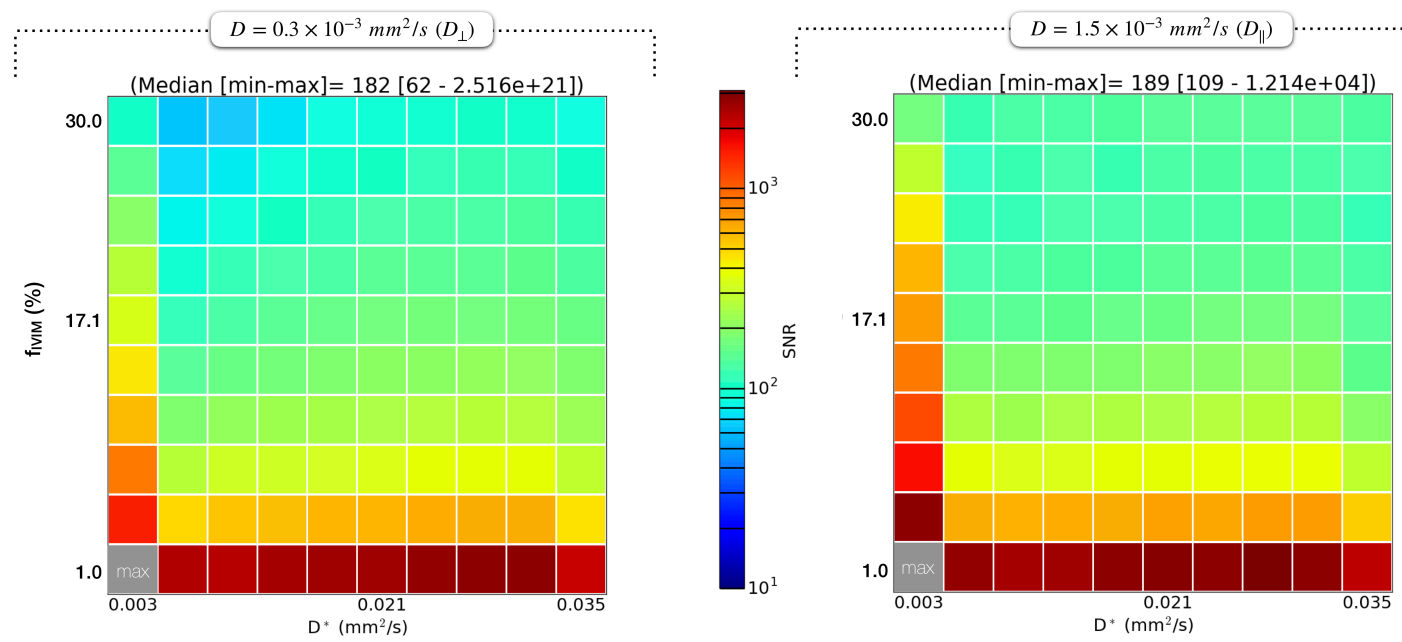

Corresponding estimation errors on parameters
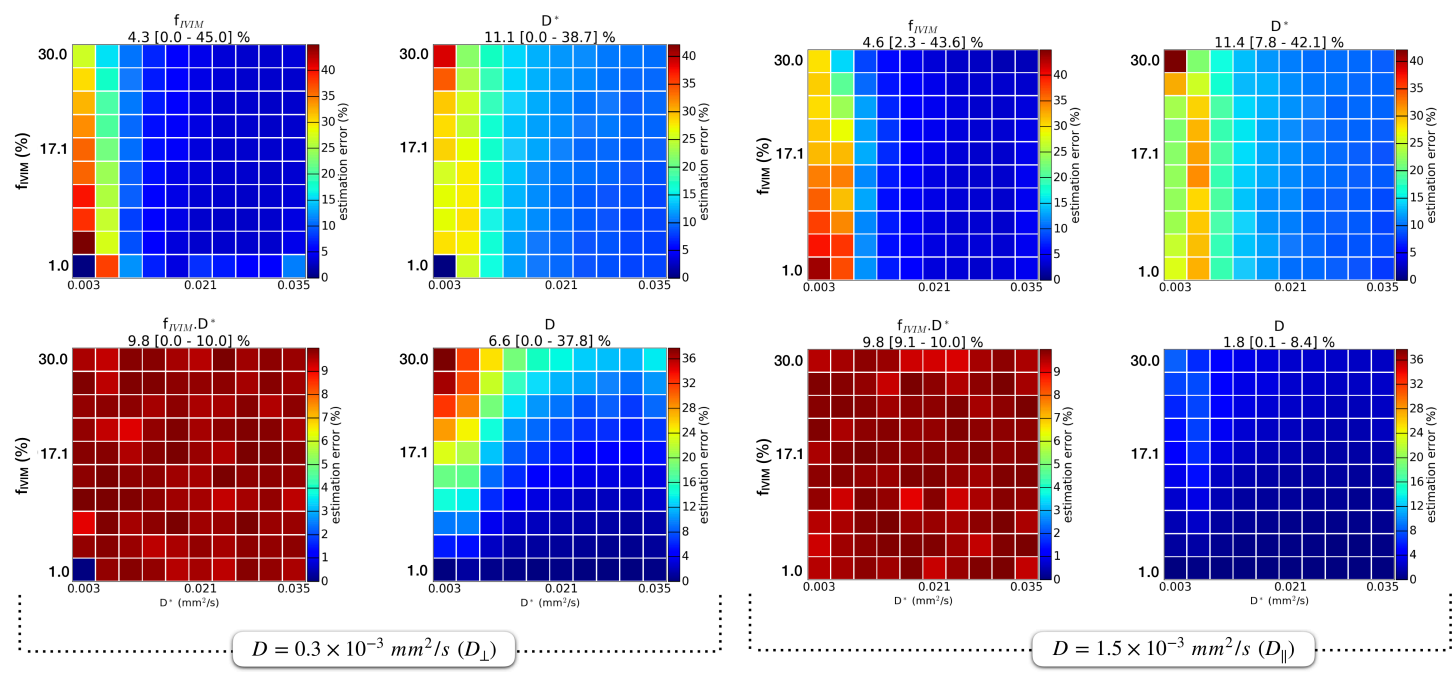

Figure 4. Top: minimum required SNR to estimate parameter $f_{I V I M} D^{*}$ with less than $10 \%$ error with the one-step fitting approach, according to $\left(f_{I V I M}, D^{*}\right)$ true values with $D=0.3 \times 10^{-3} \mathrm{~mm}^{2} / \mathrm{s}\left(D_{\perp}\right)$ and $D=1.5 \times 10^{-3} \mathrm{~mm}^{2} / \mathrm{s}\left(D_{\|}\right)$. Bottom: corresponding errors on parameters for the minimum required SNR determined above. 


\subsection{In-vivo SNR}

Figure 5 shows an example of single-subject and single-slice $b=0$ images along with the corresponding SNR, signal and noise maps at the different steps of the postprocessing pipeline. Mean SNR in SC for this particular subject and slice was $10 \pm 3$ at acquisition, $30 \pm 13$ after denoising and removal of Gibbs artefacts and $147 \pm 68$ after distortion correction and averaging across repetitions. At the group level, the minimum, mean and maximum SNR across subjects and IVIM acquisitions (diffusion-encoding directions) on the lowest $b$-value images at output of the post-processing pipeline were approximately 60, 120, 180 (see the SNR distribution of the entire cohort in Supporting Information Figure S3). Estimation errors on IVIM parameters for such SNR values are given by Figure 3.

Group-averaged maps (Figure 6-A) show that high SNR values were obtained fairly homogeneously inside the cord, generally at 1-2 voxel distance from the edge (in native space, 2-3 voxels in template space). Finally, the high image quality obtained for singlesubject and single-slice data can be observed on a representative subset of diffusionweighted images (Figure 6-C).

Note that the SNR values reported here refer to the ratio of the mean signal over the unexpected signal variations along repetitions of the same measurement as quantified by the SD across repetitions. Therefore, they represent not (only) the SNR at acquisition but refer to the SNR at the input of the fitting algorithm.

\subsection{Group averaging was necessary to discriminate between gray and white matter perfusion}

In-vivo IVIM maps are presented in Figure 7. Top images are IVIM maps obtained on a single representative subject and single slice. These maps demonstrate severe noise propagation. Mean \pm SD values within WM (resp. GM) are $14.7 \pm 8.5(21.6 \pm 9.6) \%$ for $f_{\text {IVIM }}, 7.3 \pm 5.9(3.9 \pm 3.2) \times 10^{-3} \mathrm{~mm}^{2} / \mathrm{s}$ for $D^{*}$ and $0.35 \pm 0.31(0.38 \pm 0.20) \times 10^{-3} \mathrm{~mm}^{2} / \mathrm{s}$ for $f_{I V I M} D^{*}$. These large SD values relate to large estimation errors of the fitting algorithm. Indeed, a mean voxel-wise SNR of 130 was measured within this subject's SC, which would lead to errors of $12 \%, 20 \%, 12 \%$ and $5 \%$ for $f_{I V I M}, D^{*}, f_{I V I M} D^{*}$ and $D$ respectively (according to Monte-Carlo simulations performed with $f_{I V I M}, D^{*}, D=15 \%, 11.5 \times 10^{-3}$ $\mathrm{mm}^{2} / \mathrm{s}, 0.7 \times 10^{-3} \mathrm{~mm}^{2} / \mathrm{s}$, which are the average values across subjects, Figure 8 ). These 


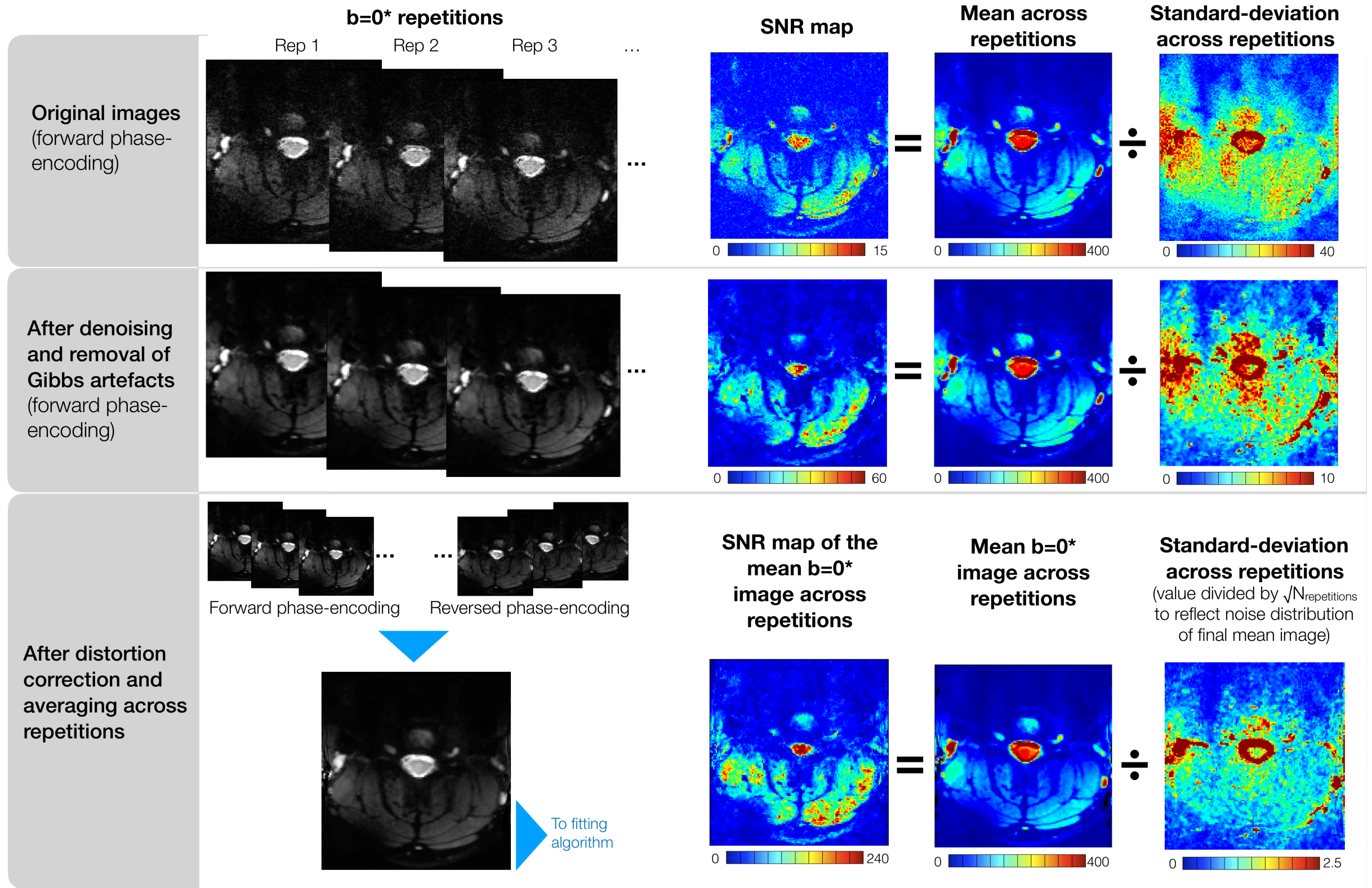

Figure 5. Representative dataset of $b=0 *$ images along the different steps of the post-processing pipeline. For each step - original images at the scanner output ( $1^{\text {st }}$ row), after denoising and removal of Gibbs ( $2^{\text {nd }}$ row $)$, after distortion correction and averaging across repetitions $\left(3^{\text {rd }}\right.$ row) - 
examples of individual repetitions, along with their SNR maps, average image across repetition and noise maps (SD across repetitions) are presented. The noise reduction along the processing steps can clearly be observed. Note that individual repetitions are rarely presented in the literature as the scanner usually performs the averaging on raw data. Processing repetitions individually before averaging is beneficial for denoising and motion correction, as evidenced by the high final image quality obtained. *Given the inherent diffusion-weighting of imaging and crusher gradients, the actual lowest b-value obtained was never 0 but $5 \mathrm{~s} / \mathrm{mm}^{2}$. 


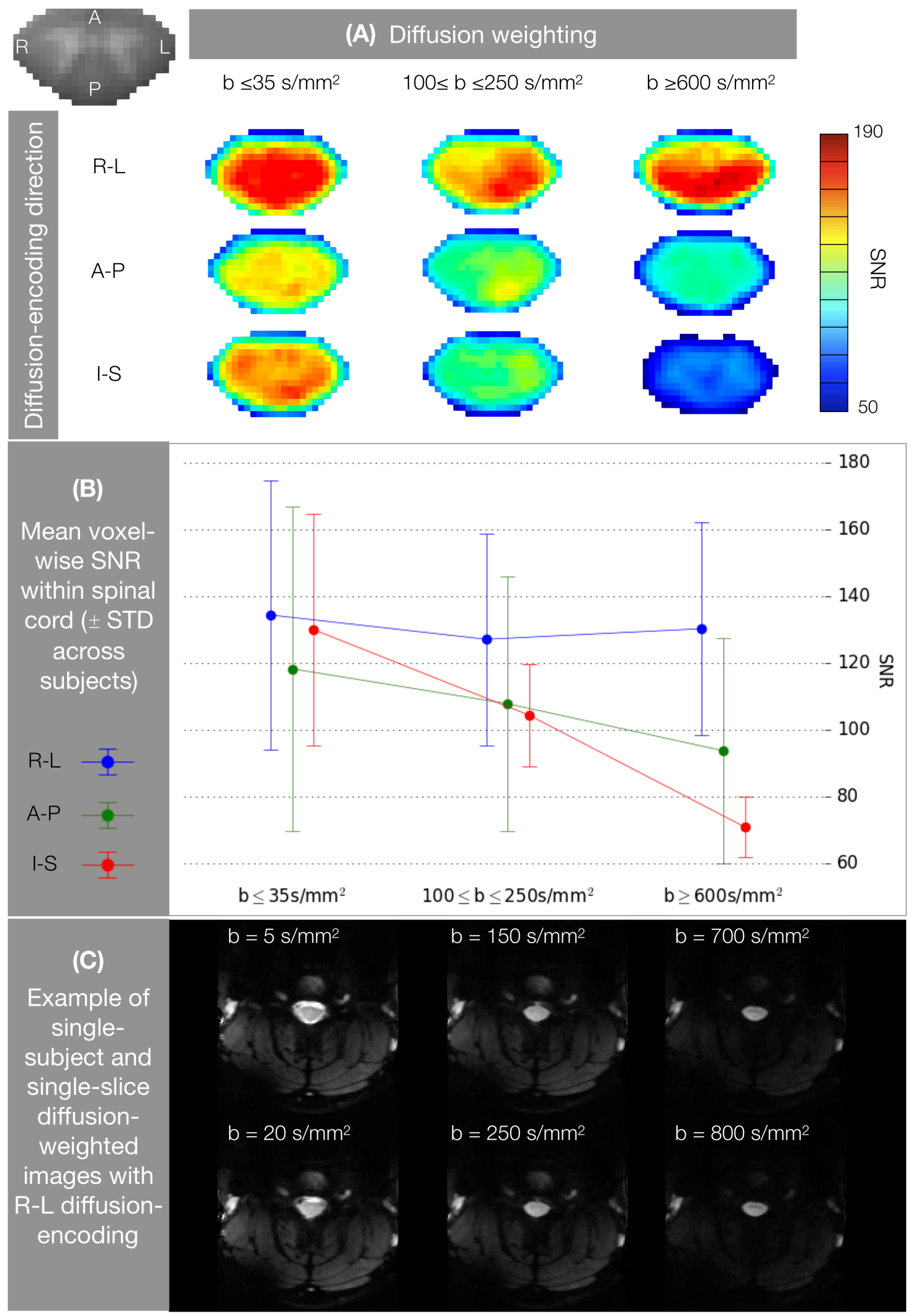

Figure 6. (A) Mean SNR maps across slices and subjects (in template space) for b-values $\leq 35 \mathrm{~s} / \mathrm{mm}^{2}$, between 100 and $250 \mathrm{~s} / \mathrm{mm}^{2}$ and $\geq 600 \mathrm{~s} / \mathrm{mm}^{2}$ for each diffusion-encoding direction: right-left (R-L, phase-encoding direction), anterior-posterior (A-P, readoutencoding direction), inferior-superior (I-S, slice-encoding direction). (B) The graph plots 
the averaged voxel-wise SNR within cord; points and error bars represent mean and SD across subjects respectively. Due to the orientation of WM fibers mainly along the SC axis, a larger SNR decrease with b-value can clearly be observed in the I-S direction than when encoding diffusion in the transverse plane (where diffusivity is much lower). Furthermore, higher SNR voxels seem to be localized in the posterior side of the cord, which is closer to the surface coil. (C) A subset of original images (6 b-values out of 11) with diffusion-encoding along R-L axis is displayed. Note the high image quality already almost depicting the GM shape. 


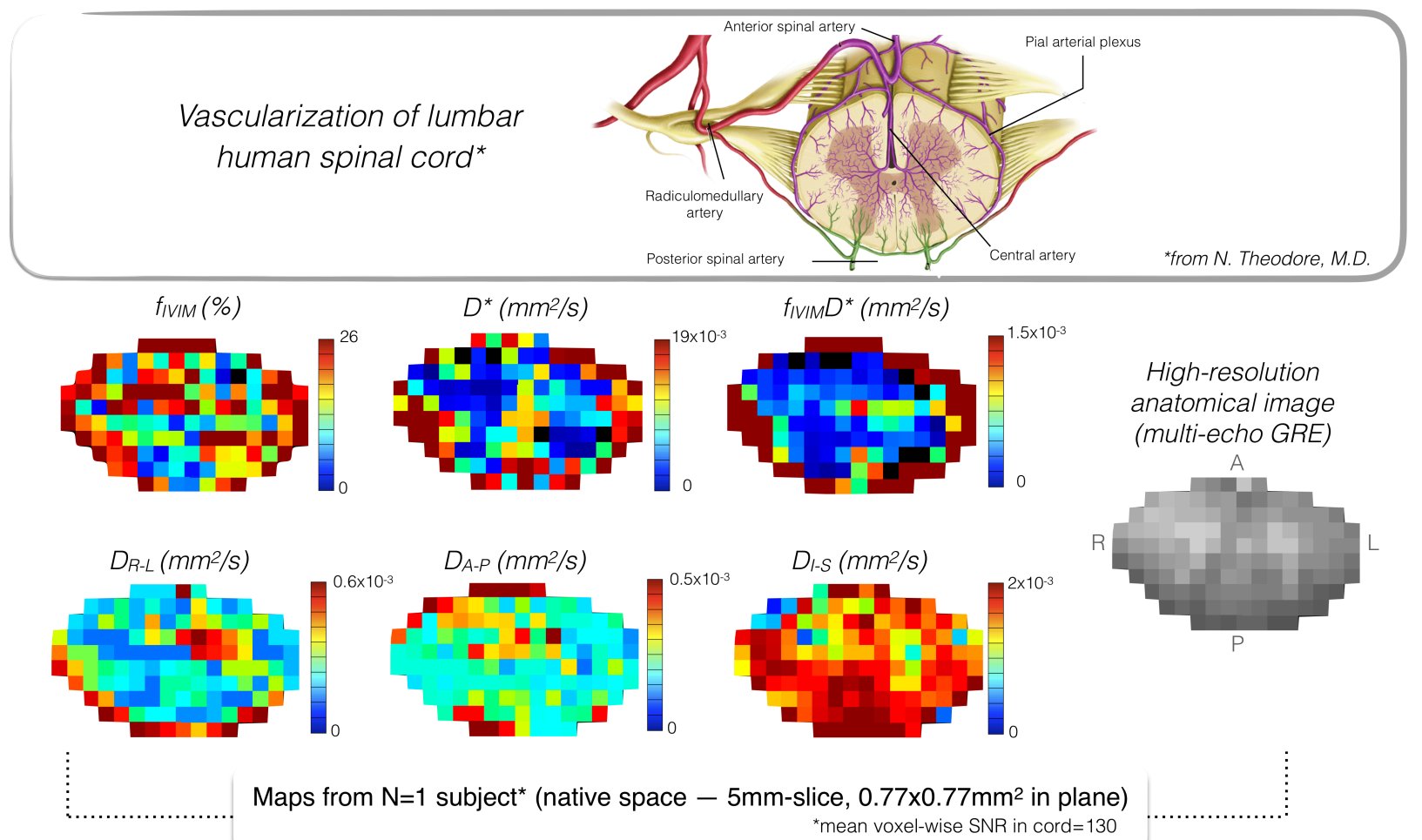

${ }^{*}$ mean voxel-wise SNR in cord $=130$
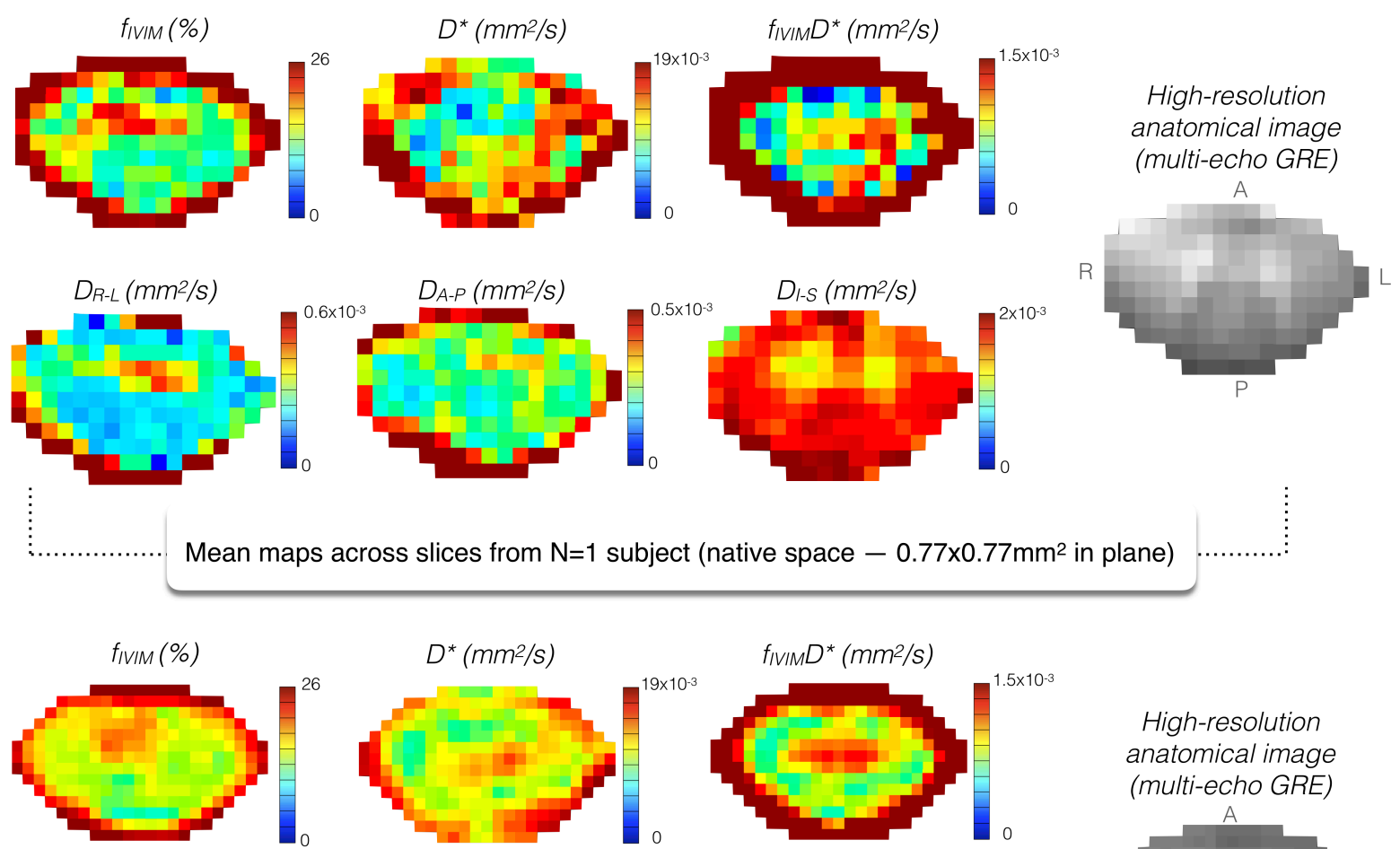

across slices from $\mathrm{N}=1$ subject (native space $-0.77 \times 0.77 \mathrm{~mm}^{2}$ in plane)
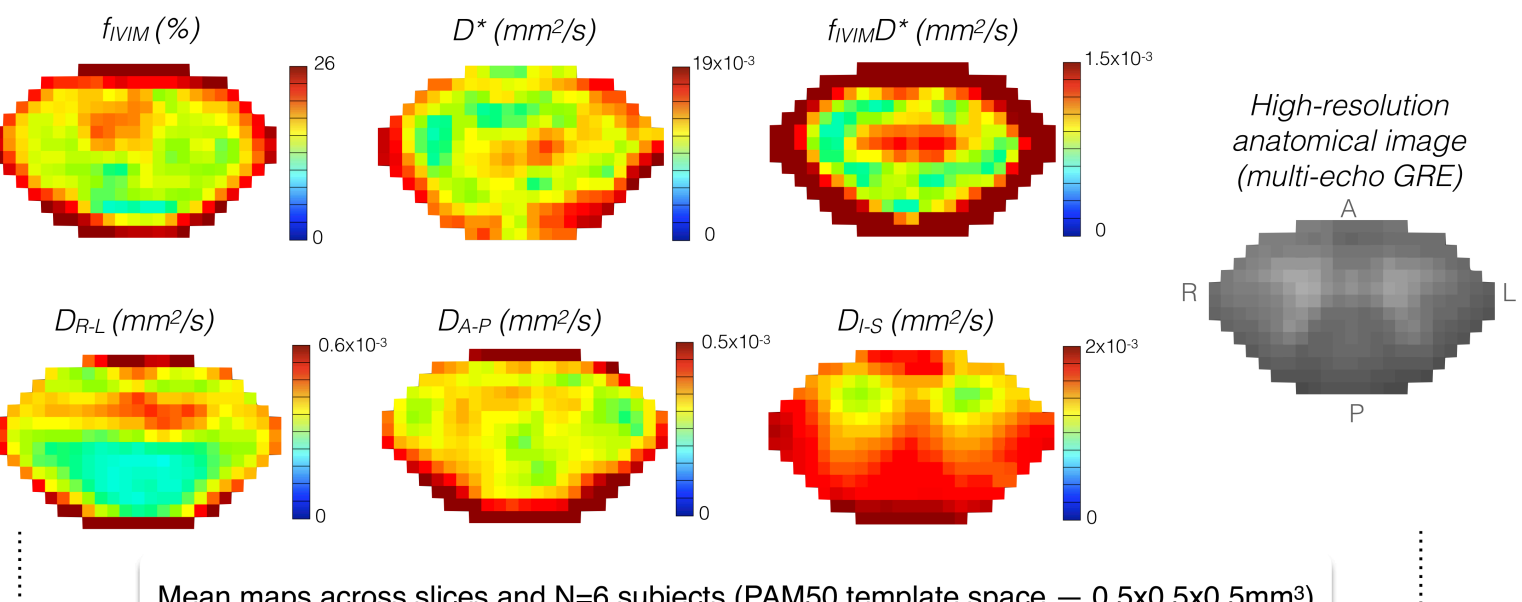

Figure 7. Spinal cord IVIM maps of a single subject (top), averaged across the six slices (middle) and averaged across slices and subjects (bottom). Slices spanned C3-C4 levels. 
High-resolution transverse anatomical images are shown alongside for visualization of GM location. All maps are the average of the three diffusion-encoding directions, except for diffusivity maps which were kept directional. $D_{R-L}, D_{A-P}$ and $D_{I-S}$ stand for diffusivity in Right-Left, Anterior-Posterior and Inferior-Superior directions. For comparison purposes, a diagram of the human spinal cord vascularization at the lumbar level (used with permissions from Nicholas Theodore, M.D.) is shown in the gray box at the top. As a result of partial volume effect with pulsatile CSF, a ring of high values about 1-2 voxelthick (in native resolution, 2-3 voxels in template resolution) can be observed at the spinal cord edge on $f_{\text {IVIM }} D^{*}$ maps. This ring is more visible after slice-averaging (second row) as one corrupted voxel in one slice would corrupt the voxel in the slice average due to the large difference in IVIM values between CSF and tissue. 
estimation margins do not allow to reliably discriminate a potential perfusion difference between GM and WM visually.

Middle row images are the average of the six slices (after nonlinear registration across slices based on MGE image) from the same subject. Mean \pm SD values within WM (resp. GM) now are $12.8 \pm 2.0(16.8 \pm 3.8) \%$ for $f_{I V I M}, 11.8 \pm 2.5(9.1 \pm 3.3) \times 10^{-3} \mathrm{~mm}^{2} / \mathrm{s}$ for $D^{*}$ and $0.88 \pm 0.31(0.83 \pm 0.28) \times 10^{-3} \mathrm{~mm}^{2} / \mathrm{s}$ for $f_{I V I M} D^{*}$. In-ROI SDs are substantially reduced. Mean coefficients of variation across slices (SD over mean across slices) within SC for this subject were $37.4 \%, 56.9 \%$ and $58.2 \%$ for $f_{I V I M}, D^{*}$ and $f_{I V I M} D^{*}$. Slice-averaging would theoretically (assuming slices are similar regarding IVIM parameters) reduced the noise in maps by a factor of $\sqrt{N_{\text {slice }}}=2.45$. A difference between GM and WM can now be visually appreciated, mainly from $f_{I V I M}$ and $f_{I V I M} D^{*}$ maps.

Bottom row images are the average of the six slices and the six subjects. Coefficients of variation across subjects were $21.1 \%, 33.6 \%$ and $39.9 \%$ for $f_{I V I M}, D^{*}$ and $f_{I V I M} D^{*}$. Values are substantially lower than before slice-averaging, showing the improved intersubject reproducibility and the common IVIM parameter distribution shared across subjects. Coefficients of variation are not exactly reduced by a factor of $\sqrt{N_{\text {slice }}}$, accounting for the inter-subject variability and potential biases in the model. The groupaveraging further reduces the noise in maps by $\sqrt{N_{\text {subjects }}}=2.45$ and eventually, subtle perfusion differences (measured at $\sim 1 \%$ of microvascular volume, Figure 8 ) can be observed between GM and WM. IVIM parameters exhibit different spatial characteristics: $f_{I V I M}$ is higher in most GM regions, $D^{*}$ is increased in the posterior part whereas $f_{I V I M} D^{*}$, a surrogate for microvascular blood flow ${ }^{47}$, comes out to be higher only in the intermediate part of GM. Furthermore, a ring of high IVIM parameters values around SC, about one voxel-thick $\left(0.77 \times 0.77 \mathrm{~mm}^{2}\right.$ in-plane in native space $)$ can be noticed both on average and individual maps. This might be due to high-velocity CSF pulsation corrupting signal in voxels at the edge of the cord but could also be attributed to the pial arterial plexus which consists of surface vessels encircling the $\mathrm{SC}^{63}$. For comparison purposes, Figure 7 also shows a diagram of the $\mathrm{SC}$ vascularization ${ }^{64}$ derived from a microangiogram of a $3 \mathrm{~mm}$ thick transverse section of human SC at lumbar level from Hassler ${ }^{65}$ (the latter study also presented a microangiogram at the $6^{\text {th }}$ thoracic level, which is closer to the cervical levels explored here). 
IVIM parameters were quantified on group- and slice-averaged maps and subsequent results are reported in Figure 8. Mean \pm SD across subjects (within-ROI) in GM and WM were $16.0 \pm 1.7$ (1.6) $\%$ and $15.0 \pm 1.6(1.7) \%$ for $f_{I V I M}, 11.4 \pm 2.9(1.4) \times 10^{-3} \mathrm{~mm}^{2} / \mathrm{s}$ and $11.5 \pm 2.4(1.4) \times 10^{-3} \mathrm{~mm}^{2} / \mathrm{s}$ for $D^{*}$, and $0.93 \pm 0.29(0.16) \times 10^{-3} \mathrm{~mm}^{2} / \mathrm{s}$ and $0.97 \pm 0.27$ $(0.23) \times 10^{-3} \mathrm{~mm}^{2} / \mathrm{s}$ for $f_{I V I M} D^{*}$. $f_{I V I M}$ suggested a slightly higher vascular volume in all GM regions compared to WM (16.0-16.5\% vs. 15.0\%), except in dorsal horns which are less vascularized than other GM regions ${ }^{64}$ and where high partial volume effects with WM could occur despite the sub-millimetric resolution. $f_{I V I M} D^{*}$ also suggested a higher microvascular flow in GM but mainly within the intermediate GM region. Estimated WM and GM pseudo-diffusion coefficients were of the same order of magnitude. Finally, $D$ values, benefiting from the lowest estimation errors $(<1 \%)$, were consistent with the underlying microstructure of each region. WM regions showed higher I-S diffusivity than GM ( 1.58 vs. $\left.1.32 \times 10^{-3} \mathrm{~mm}^{2} / \mathrm{s}\right)$ and lower radial diffusivity $\left(0.30\right.$ vs. $0.37 \times 10^{-3} \mathrm{~mm}^{2} / \mathrm{s}$ for $D_{R-L}, 0.31$ vs. $0.32 \times 10^{-3} \mathrm{~mm}^{2} / \mathrm{s}$ for $\left.D_{A-P}\right)$, in agreement with the longitudinal orientation of fibers in WM and the more isotropic microstructure of GM tissue. Furthermore, dorsal horns presented a higher $D_{A-P}$ compared to $D_{R-L}\left(0.33\right.$ vs. $\left.0.29 \times 10^{-3} \mathrm{~mm}^{2} / \mathrm{s}\right)$, reflecting fibers output along the A-P direction, while $D_{R-L}$ was predominant in anterior $(0.41 \mathrm{vs}$. $\left.0.31 \times 10^{-3} \mathrm{~mm}^{2} / \mathrm{s}\right)$ and intermediate GM (0.38 vs. $\left.0.31 \times 10^{-3} \mathrm{~mm}^{2} / \mathrm{s}\right)$, which is consistent with fibers crossing through the anterior gray commissure.

\subsection{Higher microvascular volumes and lower blood velocities along the SC axis are suggested while blood flow would be similar in all directions}

The diffusion-encoding direction strongly affects IVIM parameters values (Figure 9) as could be expected given the anisotropic structure of SC.

The measured difference in $f_{I V I M}$ values $(\geq 20 \%)$ between I-S axis and transverse plane (R-L and A-P axes) was higher than the expected errors ( $\sim 12 \%)$ based on simulations (not taking into account the noise reduction with slice- and group-averaging). However for $D^{*}$, the actual difference between I-S axis and orthogonally to the SC $(\sim 8 \%)$ was lower than estimation errors $(\sim 20 \%)$. The higher $f_{I V I M}$ and lower $D^{*}$ along the I-S axis compared to orthogonally to the cord resulted in similar $f_{I V I M} D^{*}$ values along the three axes.

Finally, relating in-vivo values to simulations in terms of SNR requirements, to estimate $f_{I V I M} D^{*}$ within $10 \%$ error margins using the implemented protocol, a minimum $\mathrm{b}=0$ SNR of 194, 156 and 137 would be needed with diffusion-encoding in R-L $\left(f_{I V I M}=12.3 \%, D^{*}=12.9 \times 10^{-3} \mathrm{~mm}^{2} / \mathrm{s}, D=0.33 \times 10^{-3} \mathrm{~mm}^{2} / \mathrm{s}\right.$ in whole SC), A-P $\left(f_{I V I M}=15.0 \%\right.$, 
$f_{\text {IVIM }}(\%)$

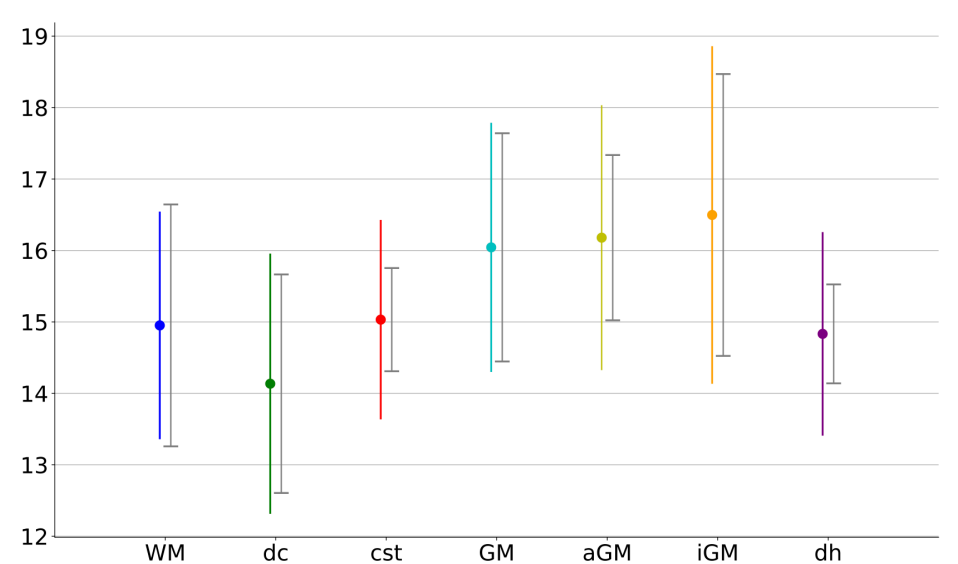

$\mathrm{f}_{\text {IVIM }} \cdot \mathrm{D}^{*}\left(\mathrm{~mm}^{2} / \mathrm{s}\right)$

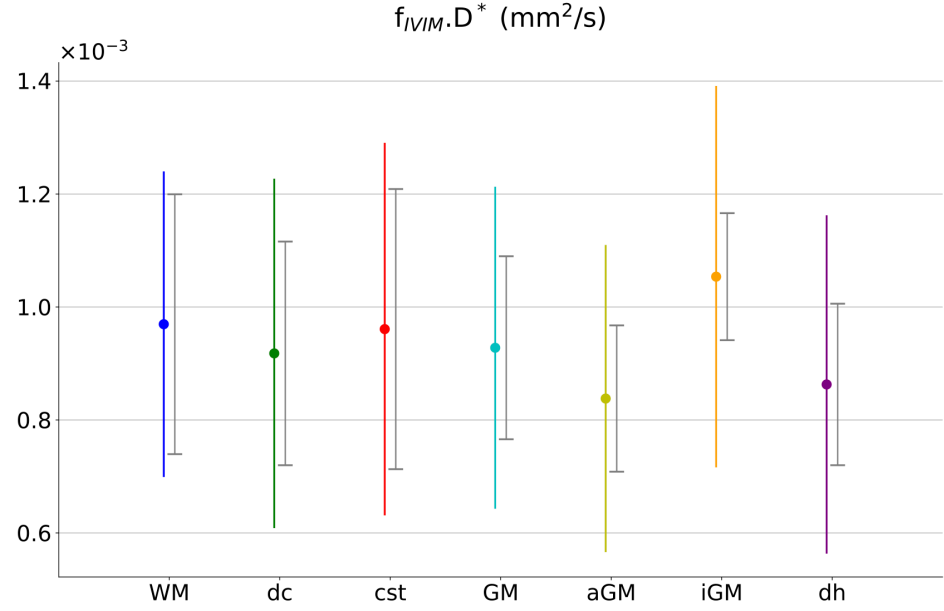

$D^{*}\left(\mathrm{~mm}^{2} / \mathrm{s}\right)$

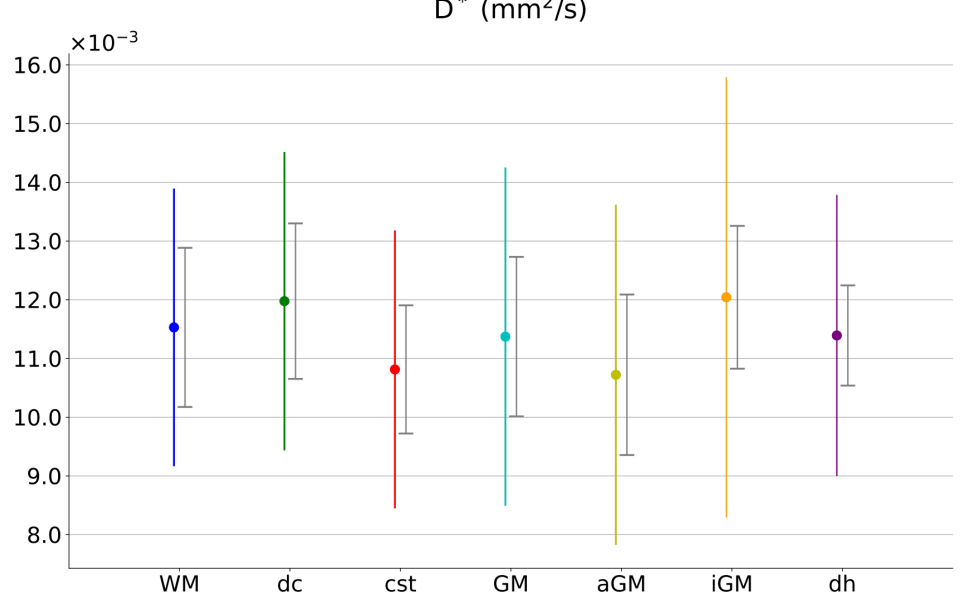

$\mathrm{D}\left(\mathrm{mm}^{2} / \mathrm{s}\right)$

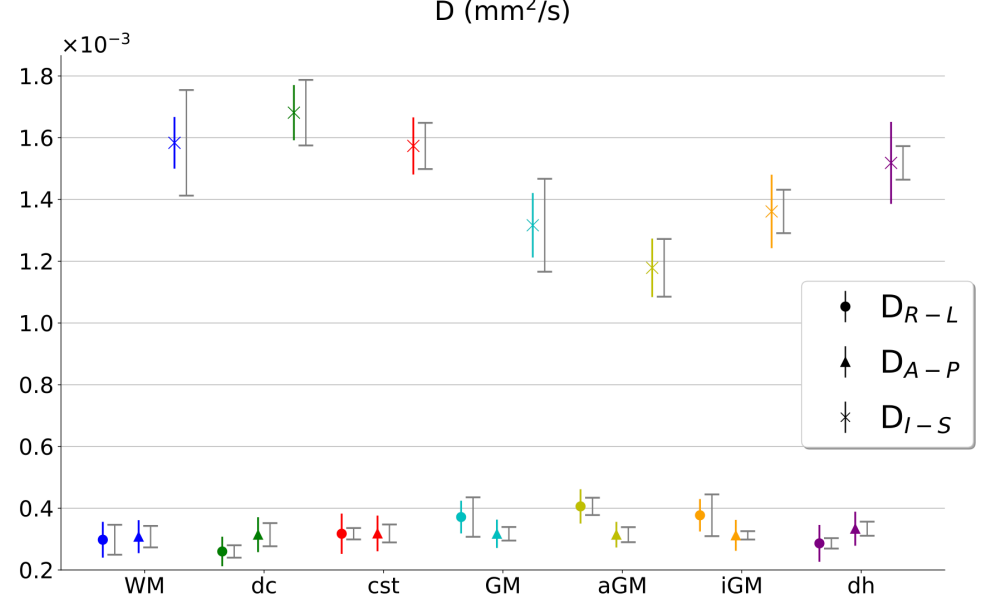

- White Matter (WM)

- dorsal columns

- Gray Matter (GM)

corticospinal tracts

anterior GM

ф intermediate GM

$\phi$ dorsal horns

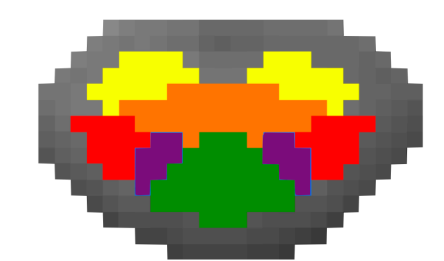

Regions of interest (ROIs) on mean multi-echo GRE

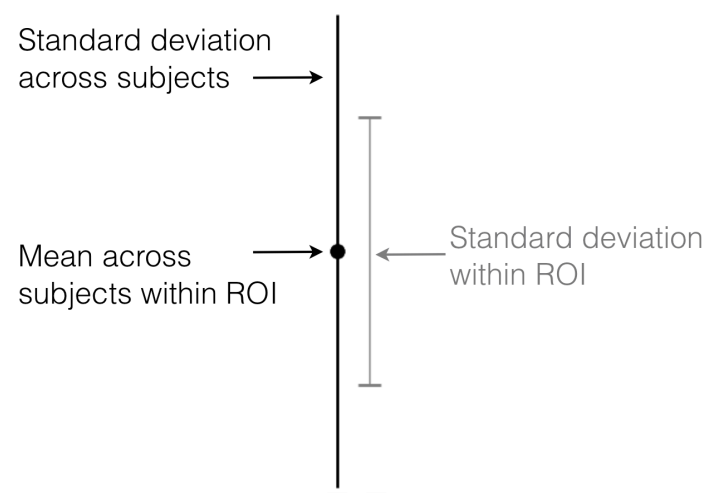

Figure 8. Parameters quantification within WM and GM ROIs. Sub-ROIs are depicted on the high-resolution MGE image: corticospinal tracts, dorsal columns (WM sub-ROIs) and anterior GM, intermediate GM, dorsal horns (GM sub-ROIs). $D_{R-\mathrm{L}}, D_{A-P}, D_{I-S}$ are the diffusion coefficients in Right-Left, Anterior-Posterior, Inferior-Superior directions respectively. 

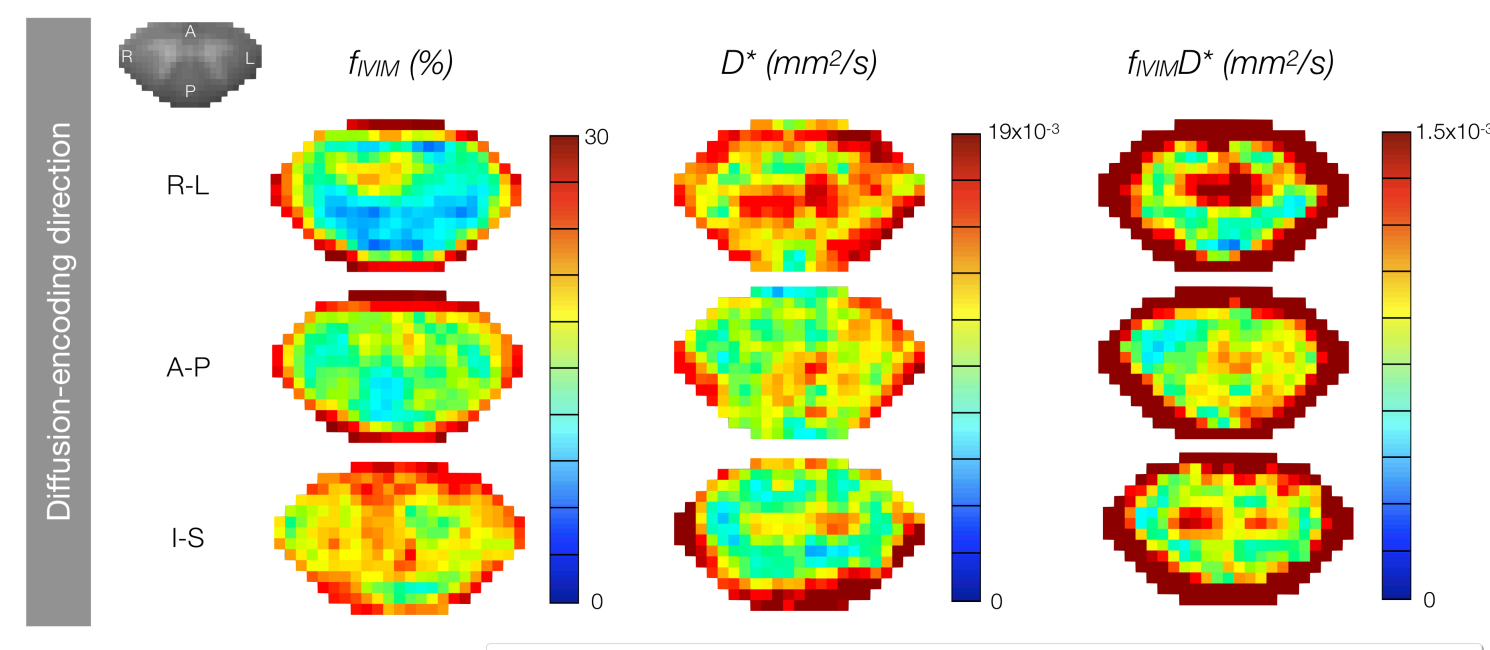

$D\left(\mathrm{~mm}^{2} / \mathrm{s}\right)$

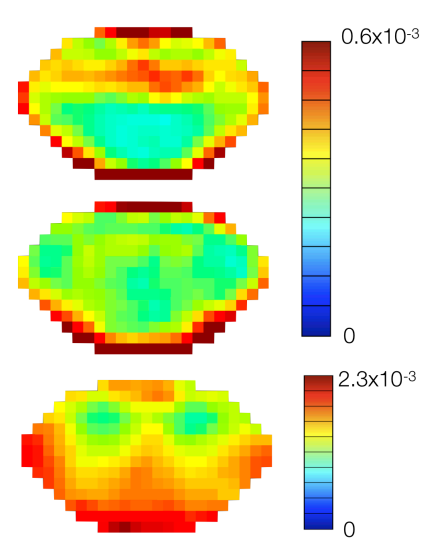

$\square$ White Matter $\square$ Gray Matter | Within-ROI standard deviation
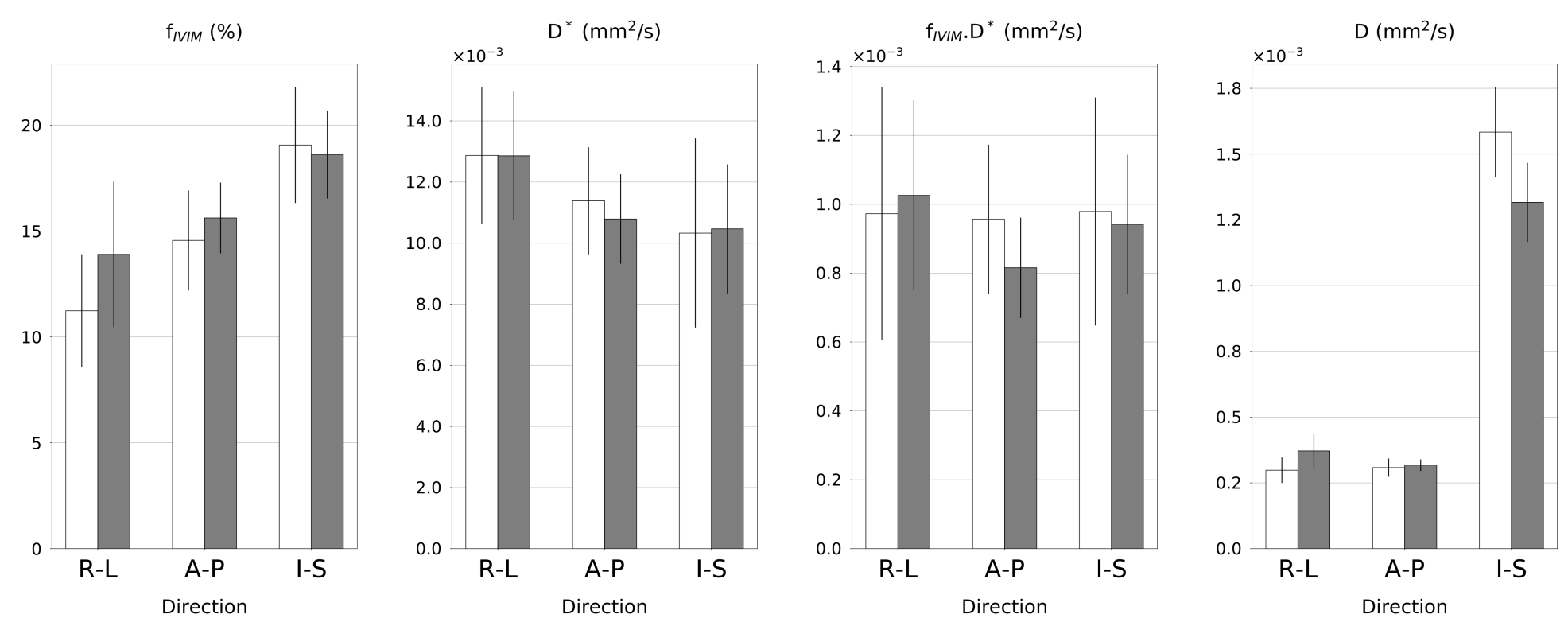

Figure 9. Directional IVIM parameter maps (mean across slices and subjects in template space, $0.5 \times 0.5 \mathrm{~mm}^{2}$ ) and quantification of IVIM parameters within GM and WM according to diffusion-encoding direction. R-L, A-P, I-S: right-left, anterior-posterior, inferior-superior directions. 
$\left.D^{*}=11.1 \times 10^{-3} \mathrm{~mm}^{2} / \mathrm{s}, \quad D=0.31 \times 10^{-3} \mathrm{~mm}^{2} / \mathrm{s}\right)$ and $\mathrm{I}-\mathrm{S} \quad\left(f_{I V I M}=18.9 \%, D^{*}=10.4 \times 10^{-3} \mathrm{~mm}^{2} / \mathrm{s}\right.$, $D=1.47 \times 10^{-3} \mathrm{~mm}^{2} / \mathrm{s}$ ) directions respectively.

\subsection{Simulations versus in-vivo data}

Figure 10 compares simulated data to in-vivo data extracted from 10 voxels in GM and 10 voxels in WM from the single-subject and single-slice maps presented in Figure 7. Similar fit quality was observed between simulations and in-vivo data (as evidenced by the coefficient of determination $R^{2}$ ), along with robust performances of the fitting algorithm. However, signal decay profiles with b-value clearly differed both between simulated and in-vivo data but also between voxels of a same tissue type for in-vivo data. In-vivo signal decay as described by the current IVIM model seems to result from more than only the IVIM effect of capillary perfusion, suggesting potential confounding factors. This also reflects in the Akaike Information Criterion corrected for sample size (AICc), which is an index of the amount of information lost by the model for the given dataset $^{66}$. The AICc was about 1.6 times higher for in-vivo data, supporting that in-vivo data included more information unexplained by the IVIM model than synthetic data which were generated from it. Finally, 3 WM in-vivo voxels stood out with a larger IVIM effect; those voxels are at the edge of the SC and were probably affected by the highvelocity pulsations of CSF. Other WM voxels showed less IVIM effect than GM voxels, as evidenced by the curve curvature. 


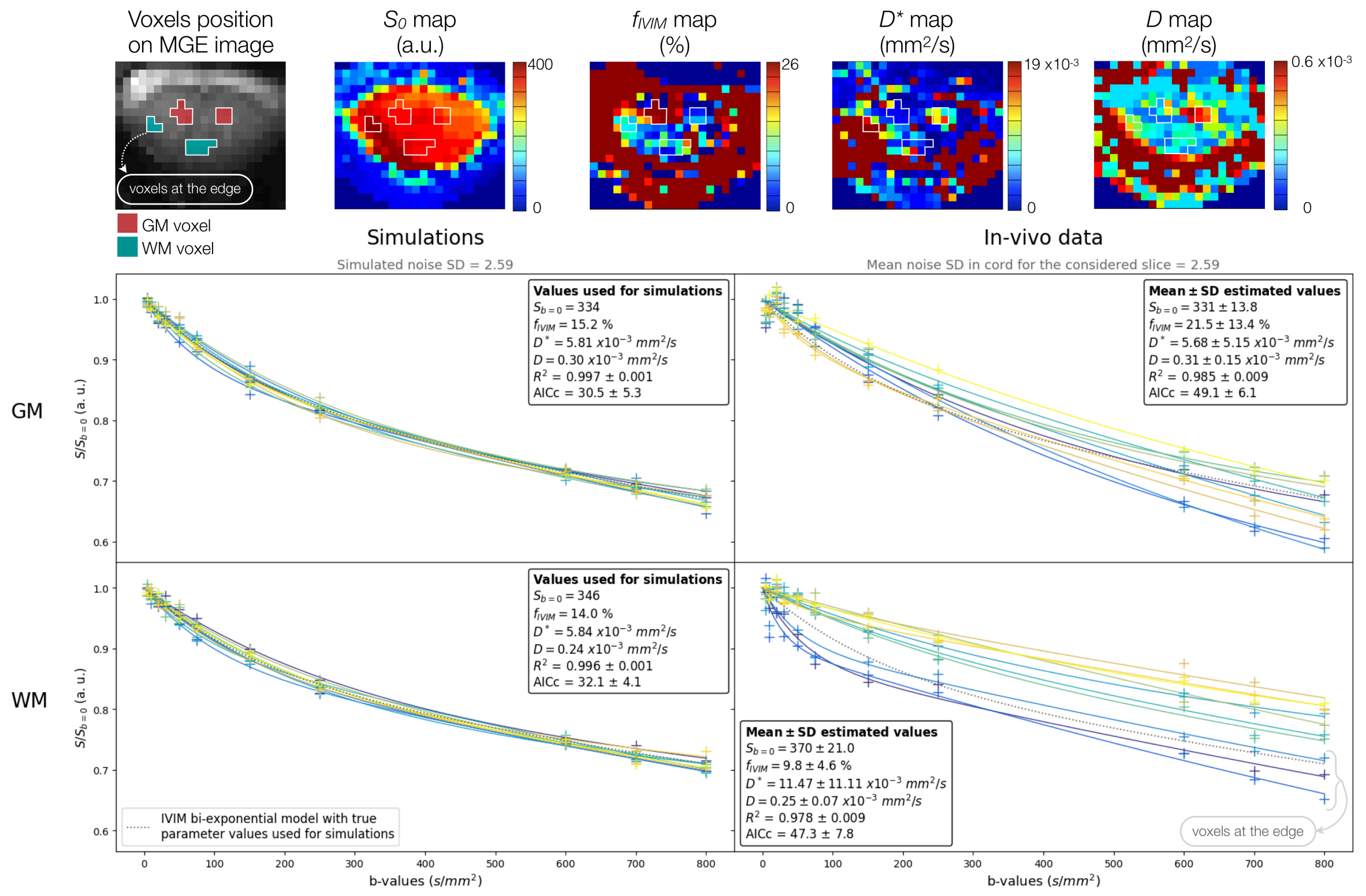

Figure 10. Simulations versus in-vivo data. On the left side, 10 voxels with the same noise level and IVIM parameters for gray and white matter were simulated and fitted. On the right side, in-vivo data from 10 voxels pseudo-randomly selected in GM (first row) and WM (second row) on 
the single-subject and single-slice IVIM maps presented in Figure 7 were fitted. All datasets were normalized by their estimated $S_{b=0}$ value. The corresponding IVIM maps were displayed at the top and the selected voxels are delineated by white borders on the maps. Selected voxels were also identified on the anatomic MGE image by color according to their tissue type (GM in red and WM in iris blue). The true IVIM parameter values for simulations were defined by the mean value in GM and WM respectively for this specific slice. Regarding in-vivo WM voxels, 3 voxels were chosen close to the SC edge; those voxels clearly exhibit larger IVIM effect than those in the dorsal column, probably due to the proximity with high-velocity pulsations of CSF. SD: standard deviation. 


\section{Discussion}

This work proposes a combined numerical and experimental study for noninvasive perfusion imaging of the human cervical SC using IVIM at 7T. Estimation errors and required SNRs were first evaluated for expected in-vivo values. IVIM maps were then derived at multiple averaging scales: from single-subject and single-slice data to the total average. The latter enabled to distinguish regional perfusion differences.

\subsection{Promises of IVIM imaging in the human SC}

While SNR within individual data was limited, averaging maps across slices and subjects provided enough SNR to highlight very small (1\% of microvascular volume fraction) regional perfusion differences between WM and GM, offering preliminary results of perfusion-related parameter mapping in the human SC in-vivo.

In this first investigative study, mean values for $f_{I V I M}$ and $D^{*}$ were $15.0 \%$ and $11.5 \times 10^{-3} \mathrm{~mm}^{2} / \mathrm{s}$ in $\mathrm{WM}$, and $16.0 \%$ and $11.4 \times 10^{-3} \mathrm{~mm}^{2} / \mathrm{s}$ in GM. Microvascular fraction values were higher than the values of $\sim 5 \%$ reported in brain using PET and ${ }^{15} \mathrm{O}$ inhalation method $^{67}$, but they are in the range of IVIM studies in normal brain GM and $\mathrm{WM}^{26,27}$ even if the cohort explored here needs to be extended. This potential overestimation can be reduced by taking into account the relaxation rate difference between blood and tissue in IVIM representation as proposed by Lemke et al. ${ }^{35}$; however this was beyond the scope of the current study. Regarding spatial distribution, also in agreement with brain studies $^{25,26,50}$ and anatomical charts ${ }^{64}$, highest microvascular volumes (on average maps) seem to match with GM location (mainly anterior and intermediate GM). Moreover, the $f_{I V I M} D^{*}$ map suggests highest flow at the location of central arteries (Figure 7) which represent the main blood supply to anterior horns ${ }^{64}$. Average IVIM maps, and especially the microvascular volume fraction $\left(f_{I V I M}\right)$, also nicely compare with the microangiogram of transverse section of human SC at T6 level from Hassler ${ }^{65}$, even though SC structure varies from thoracic to cervical levels. This microangiogram also illustrates the difficulty to capture the SC perfusion levels from such a sparse capillary network.

While our findings stem from a single-center 7T study, the protocol set up here can easily be translated to different 7T human MR sites as well as to lower clinical field with minor adaptation for potential clinical applications. Indeed, transferring the protocol to $3 \mathrm{~T}$ could benefit from lower $\mathrm{B}_{0}$ inhomogeneities, improved $\mathrm{B}_{1}$ and reduced susceptibility effects, hence mitigating the lower theoretical SNR at this field. 
Independent from field strength, our numerical evaluation also showed that there is a crucial need for standardization, and one potential asset is the growing availability of open-source programs to encourage reproducible research ${ }^{68}$. Fitting algorithms can significantly impact parameter estimation errors. In particular, some optimization algorithms (e.g., Conjugate-Gradient) showed little robustness to local optima for some parameter sets with infinite SNR whereas others, such as Differential Evolution ${ }^{48}$ (preferred here), systematically provided the global optimum of the cost function. With the objective to facilitate comparisons across studies and fitting procedures, the programs employed here were made open-source and available at https://github.com/slevyrosetti/ivim-toolbox.

We provide here the estimation errors associated with our implementation, for given SNRs. Results also depend, to some extent, on the b-value distribution but trends are consistent with those from Pekar et al. ${ }^{38}$ who used similar b-values. However, given that literature in healthy WM and GM shows variations as large as $13 \times 10^{-3} \mathrm{~mm}^{2} / \mathrm{s}$ for $D^{* 39}$ and $13 \%$ for $f_{I V I M}{ }^{26}$, the present work evaluated the full range of potential IVIM values; and indeed, results revealed a substantial variation in estimation accuracy within this range of values.

\subsection{Limitations and perspectives}

One major limitation in the current setting is that single-subject and single-slice IVIM maps were not reliable enough to clearly discriminate between GM and WM perfusion. Mean values across subjects (Figure 8) finally revealed less than $10 \%$ perfusion difference in $f_{I V I M} D^{*}$ between those two tissues while the estimation error would be at best 9\% with the highest voxel-wise in-vivo measured SNRs (180), 13\% in average $(\mathrm{SNR}=120)$ and $27 \%$ at lowest SNRs (60). This poor reliability in single-subject and single-slice maps makes the protocol currently unsuitable for detecting locally restricted abnormalities or for studying variations along the $\mathrm{SC}$ axis. So far, the best precision for an individual diagnosis would be the one obtained in slice-averaged maps. However, slice-averaging would mitigate detection of locally restricted abnormalities (e.g., existing on one slice only). Further developments are thus needed to reliably detect local perfusion abnormalities or to study variations along the SC axis.

Furthermore, the theoretical required SNR was reached in-vivo in this study but the method employed to determine estimation errors does not include inaccuracy of the IVIM biexponential model which is based on several assumptions (e.g., randomly-oriented 
microvascular network, $D^{*}>>$, no exchange between intra- and extra-vascular compartments). The comparison of simulated signal decay with b-value to in-vivo profiles (Figure 10) suggests sources of bias in the model that need to be addressed to improve estimation precision. As demonstrated in Novikov et al. ${ }^{69}$, the biexponential model is a signal representation very sensitive to biases. Potential sources of bias could be microscopic non-linear motion within the cord or interstitial fluid adding IVIM effects independent from tissue perfusion. The existence of different vessel functional pools, as introduced by several authors for IVIM in brain ${ }^{70-73}$, could also be a source of bias. Besides, the large slice thickness used in this study $(5 \mathrm{~mm})$ might mix the different effects within each voxel in a complex manner, adding to the inaccuracies. More sophisticated models have shown better accuracy ${ }^{74,75}$ but assessment of the IVIM model was beyond the scope of this article.

Still, IVIM in the SC is highly SNR-demanding as expected, and the technique as used so far will hardly be able to measure very low perfusion levels (e.g. $f_{I V I M}<4 \%$ for diffusion-encoding in the SC transverse plane, or $f_{I V I M}<8 \%$ for diffusion-encoding along the SC axis) given the required SNRs (>500) for single-subject and single-slice data. It should also be noted that the IVIM technique is very sensitive to high fluid velocities such as encountered within surrounding pulsatile CSF. This is a non-negligible issue for voxels at the edge of the $\mathrm{SC}$ where signal is highly biased by partial volume effect with $\mathrm{CSF}$, resulting in the high value ring that can be observed on $f_{I V I M}, D^{*}$ and more extensively $f_{I V I M} D^{*}$ maps. Similarly, the central canal, which contains CSF, might also be a source of bias in IVIM estimation within intermediate GM as it can widen in some pathologies such as syringomyelia ${ }^{76}$. Fluid suppression strategies with inversion recovery acquisition might be worth considering. Another avenue would be to adjust the acquisition trigger delay to subjects' cardiac cycle as the latter can vary across population, targeting the quiescent phase of both SC and CSF motion. It might also be judicious to trigger the acquisition at the peak of perfusion flow as $f_{I V I M} D^{*}$ and $D^{*}$ in brain were shown to vary across the cardiac cycle ${ }^{77}$.

Moreover, this study reports errors and required SNRs for healthy IVIM values but perfusion levels might deviate in pathological cases. In cases like gliomas where perfusion increase can go up by $+30 \times 10^{-3} \mathrm{~mm}^{2} / \mathrm{s}$ in $D^{*}$ and $+10 \%$ in $f_{I V I M}{ }^{26,41,42}$ compared to healthy values, IVIM fitting errors would be lowered down to $3 \%$ on $f_{I V I M} D^{*}$ at $\mathrm{SNR}=180$. In such cases, estimation would be precise enough to discriminate between healthy and pathological tissue. In contrast, in ischemic strokes, $f_{I V I M}$ would be reduced by 
about $-4 \%{ }^{28}$ corresponding to an error increase from $9 \%$ in healthy tissue to about $20 \%$ in ischemic tissue, making ischemia undetectable.

So far, the current acquisition protocol is too long to be used in clinical routine and might be affected by additional movements (e.g., swallowing) associated with patients' discomfort. Nonetheless, multiple avenues for improvement exist. The b-value distribution and repetition numbers were herein defined empirically; they can be optimized to reduce scan time and/or improve parameters estimation. Monte-Carlo simulations can be pushed further or the Cramer-Rao lower bounds can also be used to this purpose ${ }^{78}$. SNR increase can be achieved through multiple ways. Improved hardware like more powerful gradients or design of more efficient high-density coil arrays dedicated to ultrahigh field MRI will hopefully enable to reach higher SNR in shorter time. Alternative k-space sampling schemes such as spiral readout and inner FOV sequences would reduce TE and distortions associated with single-shot EPI. Finally, cutting-edge fitting approaches might be worth considering. For instance, Bayesian methods - which basically maximize the probability density function of parameters showed more robustness to noise $\mathrm{e}^{79,80}$, but caution has to be taken as fine perfusion variations could be smoothed when parameter uncertainty is high ${ }^{81}$.

Nonetheless, the IVIM technique was able to provide us with the first perfusionrelated maps of the human spinal cord in-vivo without contrast agent injection, alleviating concerns regarding the use of several gadolinium chelates ${ }^{15,16}$. In addition, along with perfusion information, IVIM provides microstructural information from the diffusion coefficient, which can be useful in the joint assessment of perfusion and tissue integrity in the context of SC injuries. 


\section{Conclusion}

This study ultimately provides, to our knowledge, the first perfusion-related maps of the human SC, paving the way to in-vivo study of SC micro-vascularization processes, for earlier diagnostic of perfusion abnormalities in a more distant future. Ultrahigh field MRI acquisition developments and numerical simulations were combined to quantify IVIM parameters and related estimation errors for representative in-vivo SNRs. Although the technique did not show sufficient reliability to achieve patient- and level-specific IVIM mapping, the developed method provided preliminary results of SC perfusion mapping highlighting a very small perfusion difference between GM and WM $(\sim 1 \%$ of microvascular volume fraction) on group-averaged maps. Developments to increase SNR so as to reduce scan time and to address potential modeling biases are warranted. 


\section{Acknowledgements}

The authors would like to sincerely thank Ludovic de Rochefort, Olivier Girard and Sylviane Confort-Gouny for useful discussions, as well as Christophe Vilmen and Lauriane Pini for the study logistics.

This project received funding from the European Union's Horizon 2020 research and innovation program under the Marie Skłodowska-Curie grant agreement \#713750. Also, it was carried out with the financial support of the Regional Council of Provence-AlpesCôte d'Azur and with the financial support of the A*MIDEX (\#ANR-11-IDEX-0001-02), funded by the Investissements d'Avenir project funded by the French Government, managed by the French National Research Agency (ANR).

This work was performed within a laboratory member of France Life Imaging network (grant ANR-11-INBS-0006), supported by the following funding sources: 7T-AMI-ANR11-EQPX-0001, A*MIDEX-EI-13-07-130115-08.38-7T-AMISTART and CNRS (Centre National de la Recherche Scientifique). 


\section{References}

1. Kolenda H, Steffens H, Gefeller O, Hagenah J, Schomburg E. Critical levels of spinal cord blood flow and duration of ischemia for the acute recovery of segmental spinal cord responses in cats. J Spinal Disord. 1997;10(4):288-295.

2. Fehlings MG, Tetreault L, Hsieh PC, Traynelis V, Wang MY. Introduction: Degenerative cervical myelopathy: diagnostic, assessment, and management strategies, surgical complications, and outcome prediction. Neurosurg Focus. 2016;40(6):E1. doi:10.3171/2016.3.FOCUS16111

3. Vilaça CDO, Orsini M, Araujo Leite MA, et al. Cervical spondylotic myelopathy: what the neurologist should know. Neurol Int. 2016;8(4). doi:10.4081/ni.2016.6330

4. Martin AR, De Leener B, Cohen-Adad J, et al. Monitoring for myelopathic progression with multiparametric quantitative MRI. Toft M, ed. PLOS ONE. 2018;13(4):e0195733. doi:10.1371/journal.pone.0195733

5. Martin AR, Aleksanderek I, Cohen-Adad J, et al. Translating state-of-the-art spinal cord MRI techniques to clinical use: A systematic review of clinical studies utilizing DTI, MT, MWF, MRS, and fMRI. NeuroImage Clin. 2016;10:192-238. doi:10.1016/j.nicl.2015.11.019

6. Fradet L, Arnoux P-J, Ranjeva J-P, Petit Y, Callot V. Morphometrics of the Entire Human Spinal Cord and Spinal Canal Measured From In Vivo High-Resolution Anatomical Magnetic Resonance Imaging: Spine. 2014;39(4):E262-E269. doi:10.1097/BRS.0000000000000125

7. Figley CR, Stroman PW. Investigation of human cervical and upper thoracic spinal cord motion: Implications for imaging spinal cord structure and function. Magn Reson Med. 2007;58(1):185-189. doi:10.1002/mrm.21260

8. Duhamel G, Callot V, Cozzone PJ, Kober F. Spinal cord blood flow measurement by arterial spin labeling. Magn Reson Med. 2008;59:846-854. doi:10.1002/mrm.21567

9. Martirosian P, Klose U, Mader I, Schick F. FAIR true-FISP perfusion imaging of the kidneys. Magn Reson Med. 2004;51(2):353-361. doi:10.1002/mrm.10709

10. Leporq B, Saint-Jalmes H, Rabrait C, et al. Optimization of intra-voxel incoherent motion imaging at 3.0 Tesla for fast liver examination: Optimization of Liver Motion Imaging at 3.0T. J Magn Reson Imaging. 2015;41(5):1209-1217. doi:10.1002/jmri.24693

11. Wang C, Han X, Jiang W, et al. Perfusion of Spinal Cord in postoperative patient with Cervical Spondylotic Myelopathy using MR DSC technique. In: Proceedings of the 25th Annual Meeting of the International Society for Magnetic Resonance in Medicine. Honolulu, Hawai, USA; 2017:2507.

12. Wang C, Han X, Jiang W, et al. Spinal Cord Perfusion is Associated with Diffusion and clinical mJOA score in Preoperative Patients with Cervical Spondylotic 
Myelopathy. In: Proceedings of the 26th Annual Meeting of the International Society for Magnetic Resonance in Medicine. Paris, France; 2018:5397.

13. Ellingson BM, Woodworth DC, Leu K, Salamon N, Holly LT. Spinal cord perfusion MR imaging implicates both ischemia and hypoxia in the pathogenesis of cervical spondylosis. World Neurosurg. 2019. doi:10.1016/j.wneu.2019.04.253

14. Cuvinciuc V, Viallon M, Barnaure I, Vargas MI, Lovblad K-O, Haller S. Dynamic Contrast-Enhanced MR Perfusion of Intradural Spinal Lesions. Am J Neuroradiol. 2017;38:192-194. doi:10.3174/ajnr.A4995

15. Ramalho J, Semelka RC, Ramalho M, Nunes RH, AlObaidy M, Castillo M. Gadolinium-Based Contrast Agent Accumulation and Toxicity: An Update. Am J Neuroradiol. 2016;37(7):1192-1198. doi:10.3174/ajnr.A4615

16. Kanda T, Fukusato T, Matsuda M, et al. Gadolinium-based Contrast Agent Accumulates in the Brain Even in Subjects without Severe Renal Dysfunction: Evaluation of Autopsy Brain Specimens with Inductively Coupled Plasma Mass Spectroscopy. Radiology. 2015;276(1):228-232. doi:10.1148/radiol.2015142690

17. Alsop DC, Detre JA, Golay X, et al. Recommended implementation of arterial spinlabeled perfusion MRI for clinical applications: A consensus of the ISMRM perfusion study group and the European consortium for ASL in dementia: Recommended Implementation of ASL for Clinical Applications. Magn Reson Med. 2015;73(1):102-116. doi:10.1002/mrm.25197

18. Duhamel G, Callot V, Decherchi P, et al. Mouse lumbar and cervical spinal cord blood flow measurements by arterial spin labeling: Sensitivity optimization and first application. Magn Reson Med. 2009;62:430-439. doi:10.1002/mrm.22015

19. Girard OM, Callot V, Robert B, Cozzone PJ, Duhamel G. Perfusion MRI of the Human Cervical Spinal Cord using Arterial Spin Labeling. In: Proceedings of the 21st Annual Meeting of the International Society for Magnetic Resonance in Medicine. Salt Lake City, Utah, USA; 2013:0349.

20. Nair G, Hu XP. Perfusion Imaging of the Human Cervical Spinal Cord. In: Proceedings of the 19th Annual Meeting of the International Society for Magnetic Resonance in Medicine. Stockholm, Sweden; 2010:4083.

21. Wang Y, Moeller S, Li X, et al. Simultaneous multi-slice Turbo-FLASH imaging with CAIPIRINHA for whole brain distortion-free pseudo-continuous arterial spin labeling at 3 and 7T. NeuroImage. 2015;113:279-288.

doi:10.1016/j.neuroimage.2015.03.060

22. Massire A, Taso M, Besson P, Guye M, Ranjeva J-P, Callot V. High-resolution multiparametric quantitative magnetic resonance imaging of the human cervical spinal cord at 7T. NeuroImage. 2016;143:58-69. doi:10.1016/j.neuroimage.2016.08.055

23. Massire A, Rasoanandrianina H, Taso M, et al. Feasibility of single-shot multi-level multi-angle diffusion tensor imaging of the human cervical spinal cord at 7T. Magn Reson Med. 2018;80(3):947-957. doi:10.1002/mrm.27087 
24. Le Bihan D, Breton E, Lallemand D, Aubin ML, Vignaud J, Laval-Jeantet M. Separation of diffusion and perfusion in intravoxel incoherent motion MR imaging. Radiology. 1988;168(2):497-505. doi:10.1148/radiology.168.2.3393671

25. Wang C, Ren D, Guo Y, et al. Distribution of intravoxel incoherent motion MRIrelated parameters in the brain: evidence of interhemispheric asymmetry. Clin Radiol. 2017;72(1):94.e1-94.e6. doi:10.1016/j.crad.2016.09.007

26. Bisdas S, Klose U. IVIM analysis of brain tumors: an investigation of the relaxation effects of CSF, blood, and tumor tissue on the estimated perfusion fraction. Magn Reson Mater Phys Biol Med. 2015;28(4):377-383. doi:10.1007/s10334-014-0474-z

27. Bertleff M, Domsch S, Weingärtner S, et al. Diffusion parameter mapping with the combined intravoxel incoherent motion and kurtosis model using artificial neural networks at 3 T. NMR Biomed. 2017;30(12):e3833. doi:10.1002/nbm.3833

28. Suo S, Cao M, Zhu W, et al. Stroke assessment with intravoxel incoherent motion diffusion-weighted MRI. NMR Biomed. 2016;29(3):320-328. doi:10.1002/nbm.3467

29. Federau C. Intravoxel incoherent motion MRI as a means to measure in vivo perfusion: A review of the evidence. NMR Biomed. 2017;30(11):e3780. doi:10.1002/nbm.3780

30. Maximov II, Vellmer S. Isotropically weighted intravoxel incoherent motion brain imaging at 7T. Magn Reson Imaging. 2019;57:124-132. doi:10.1016/j.mri.2018.11.007

31. Milani B, Ledoux J-B, Rotzinger DC, et al. Image acquisition for intravoxel incoherent motion imaging of kidneys should be triggered at the instant of maximum blood velocity: evidence obtained with simulations and in vivo experiments. Magn Reson Med. 2019;81(1):583-593. doi:10.1002/mrm.27393

32. Gambarota G, Hitti E, Leporq B, Saint-Jalmes H, Beuf O. Eliminating the blood-flow confounding effect in intravoxel incoherent motion (IVIM) using the non-negative least square analysis in liver: NNLS Analysis of IVIM Data in Liver. Magn Reson Med. 2017;77(1):310-317. doi:10.1002/mrm.26085

33. Jalnefjord O, Andersson M, Montelius M, et al. Comparison of methods for estimation of the intravoxel incoherent motion (IVIM) diffusion coefficient (D) and perfusion fraction (f). Magn Reson Mater Phys Biol Med. August 2018. doi:10.1007/s10334-018-0697-5

34. Xiang Z, Ai Z, Liang J, Li G, Zhu X, Yan X. Evaluation of Regional Variability and Measurement Reproducibility of Intravoxel Incoherent Motion Diffusion Weighted Imaging Using a Cardiac Stationary Phase Based ECG Trigger Method. BioMed Res Int. 2018;2018:1-11. doi:10.1155/2018/4604218

35. Lemke A, Laun FB, Simon D, Stieltjes B, Schad LR. An in vivo verification of the intravoxel incoherent motion effect in diffusion-weighted imaging of the abdomen. Magn Reson Med. 2010;64(6):1580-1585. doi:10.1002/mrm.22565 
36. Barbieri S, Donati OF, Froehlich JM, Thoeny HC. Impact of the calculation algorithm on biexponential fitting of diffusion-weighted MRI in upper abdominal organs. Magn Reson Med. 2016;75(5):2175-2184. doi:10.1002/mrm.25765

37. Gurney-Champion OJ, Klaassen R, Froeling M, et al. Comparison of six fit algorithms for the intra-voxel incoherent motion model of diffusion-weighted magnetic resonance imaging data of pancreatic cancer patients. PLOS ONE. 2018;13(4):e0194590. doi:10.1371/journal.pone.0194590

38. Pekar J, Moonen CTW, van Zijl PCM. On the precision of diffusion/perfusion imaging by gradient sensitization. Magn Reson Med. 1992;23(1):122-129. doi:10.1002/mrm.1910230113

39. Federau C, O’Brien K, Meuli R, Hagmann P, Maeder P. Measuring brain perfusion with intravoxel incoherent motion (IVIM): Initial clinical experience. J Magn Reson Imaging. 2014;39(3):624-632. doi:10.1002/jmri.24195

40. Federau C, Maeder P, O’Brien K, Browaeys P, Meuli R, Hagmann P. Quantitative Measurement of Brain Perfusion with Intravoxel Incoherent Motion MR Imaging. Radiology. 2012;265(3):874-881. doi:10.1148/radiol.12120584

41. Bisdas S, Koh TS, Roder C, et al. Intravoxel incoherent motion diffusion-weighted MR imaging of gliomas: feasibility of the method and initial results. Neuroradiology. 2013;55(10):1189-1196. doi:10.1007/s00234-013-1229-7

42. Federau C, Meuli R, O’Brien K, Maeder P, Hagmann P. Perfusion Measurement in Brain Gliomas with Intravoxel Incoherent Motion MRI. Am J Neuroradiol. 2014;35(2):256-262. doi:10.3174/ajnr.A3686

43. Taso M, Girard OM, Duhamel G, et al. Tract-specific and age-related variations of the spinal cord microstructure: a multi-parametric MRI study using diffusion tensor imaging (DTI) and inhomogeneous magnetization transfer (ihMT). NMR Biomed. 2016;29:817-832. doi:10.1002/nbm.3530

44. Finkenstaedt T, Klarhoefer M, Eberhardt C, et al. The IVIM signal in the healthy cerebral gray matter: A play of spherical and non-spherical components. NeuroImage. 2017;152:340-347. doi:10.1016/j.neuroimage.2017.03.004

45. Samson RS, Lévy S, Schneider T, et al. ZOOM or Non-ZOOM? Assessing Spinal Cord Diffusion Tensor Imaging Protocols for Multi-Centre Studies. PLOS ONE. 2016;11:e0155557. doi:10.1371/journal.pone.0155557

46. Xu J, Shimony JS, Klawiter EC, et al. Improved in vivo diffusion tensor imaging of human cervical spinal cord. NeuroImage. 2013;67:64-76. doi:10.1016/j.neuroimage.2012.11.014

47. Bihan DL, Turner R. The capillary network: a link between ivim and classical perfusion. Magn Reson Med. 1992;27(1):171-178. doi:10.1002/mrm.1910270116

48. Storn R, Price K. Differential Evolution - A Simple and Efficient Heuristic for global Optimization over Continuous Spaces. J Glob Optim. 1997;11(4):341-359. doi:10.1023/A:1008202821328 
49. Vonken EPhA, van Osch MJP, Bakker CJG, Viergever MA. Measurement of cerebral perfusion with dual-echo multi-slice quantitative dynamic susceptibility contrast MRI. J Magn Reson Imaging. 1999;10(2):109-117. doi:10.1002/(SICI)15222586(199908)10:2<109::AID-JMRI1>3.0.CO;2-\#

50. Parkes Laura M., Rashid Waqar, Chard Declan T., Tofts Paul S. Normal cerebral perfusion measurements using arterial spin labeling: Reproducibility, stability, and age and gender effects. Magn Reson Med. 2004;51(4):736-743.

doi: $10.1002 / \mathrm{mrm} .20023$

51. Morelli JN, Runge VM, Feiweier T, Kirsch JE, Williams KW, Attenberger UI. Evaluation of a modified Stejskal-Tanner diffusion encoding scheme, permitting a marked reduction in TE, in diffusion-weighted imaging of stroke patients at $3 \mathrm{~T}$. Invest Radiol. 2010;45(1):29-35. doi:10.1097/RLI.0b013e3181c65c11

52. DiIorio G, Brown JJ, Borrello JA, Perman WH, Shu HH. Large angle spin-echo imaging. Magn Reson Imaging. 1995;13(1):39-44. doi:10.1016/0730725X(94)00082-E

53. Alley S, Gilbert G, Gandini Wheeler-Kingshott C, et al. Consensus acquisition protocol for quantitative MRI of the cervical spinal cord at 3T. In: Proceedings of the 26th Annual Meeting of the International Society for Magnetic Resonance in Medicine. Paris, France; 2018:0799.

54. Manjón JV, Coupé P, Concha L, Buades A, Collins DL, Robles M. Diffusion Weighted Image Denoising Using Overcomplete Local PCA. PLOS ONE. 2013;8(9):e73021. doi:10.1371/journal.pone.0073021

55. Kellner E, Dhital B, Kiselev VG, Reisert M. Gibbs-ringing artifact removal based on local subvoxel-shifts. Magn Reson Med. 2016;76(5):1574-1581. doi: $10.1002 / \mathrm{mrm} .26054$

56. De Leener B, Lévy S, Dupont SM, et al. SCT: Spinal Cord Toolbox, an open-source software for processing spinal cord MRI data. NeuroImage. 2017;145:24-43. doi:10.1016/j.neuroimage.2016.10.009

57. Andersson JLR, Skare S, Ashburner J. How to correct susceptibility distortions in spin-echo echo-planar images: application to diffusion tensor imaging. NeuroImage. 2003;20(2):870-888. doi:10.1016/S1053-8119(03)00336-7

58. Reeder SB, Wintersperger BJ, Dietrich O, et al. Practical approaches to the evaluation of signal-to-noise ratio performance with parallel imaging: Application with cardiac imaging and a 32-channel cardiac coil. Magn Reson Med. 2005;54(3):748-754. doi: $10.1002 / \mathrm{mrm} .20636$

59. De Leener B, Fonov VS, Collins DL, Callot V, Stikov N, Cohen-Adad J. PAM50: Unbiased multimodal template of the brainstem and spinal cord aligned with the ICBM152 space. NeuroImage. 2018;165:170-179. doi:10.1016/j.neuroimage.2017.10.041 
60. Lévy S, Benhamou M, Naaman C, Rainville P, Callot V, Cohen-Adad J. White matter atlas of the human spinal cord with estimation of partial volume effect. NeuroImage. 2015;119:262-271. doi:10.1016/j.neuroimage.2015.06.040

61. De Leener B, Kadoury S, Cohen-Adad J. Robust, accurate and fast automatic segmentation of the spinal cord. NeuroImage. 2014;98:528-536.

doi:10.1016/j.neuroimage.2014.04.051

62. Perone CS, Calabrese E, Cohen-Adad J. Spinal cord gray matter segmentation using deep dilated convolutions. Sci Rep. 2018;8(1):5966. doi:10.1038/s41598-018-243043

63. Gillilan LA. The arterial blood supply of the human spinal cord. J Comp Neurol. 1958;110(1):75-103. doi:10.1002/cne.901100104

64. Martirosyan NL, Feuerstein JS, Theodore N, Cavalcanti DD, Spetzler RF, Preul MC. Blood supply and vascular reactivity of the spinal cord under normal and pathological conditions: a review. J Neurosurg Spine. 2011;15(3):238-251.

65. Hassler O. Blood supply to human spinal cord: a microangiographic study. Arch Neurol. 1966;15(3):302-307.

66. Akaike H. A new look at the statistical model identification. IEEE Transactions on Automatic Control. 1974;19(6):716-723.

67. Lammertsma AA, Rhodes C, Perani D, et al. Cerebral blood flow, blood volume and oxygen utilization: normal values and effect of age. Brain. 1990;113(1):27-47. doi:10.1093/brain/113.1.27

68. Stikov N, Trzasko JD, Bernstein MA. Reproducibility and the future of MRI research. Magn Reson Med. August 2019:mrm.27939. doi:10.1002/mrm.27939

69. Novikov DS, Kiselev VG, Jespersen SN. On modeling. Magn Reson Med. 2018;79(6):3172-3193. doi:10.1002/mrm.27101

70. Kennan RP, Gao J-H, Zhong J, Gore JC. A general model of microcirculatory blood flow effects in gradient sensitized MRI. Med Phys. 1994;21(4):539-545. doi:10.1118/1.597170

71. Henkelman RM, Neil JJ, Xiang Q-S. A quantitative interpretation of IVIM measurements of vascular perfusion in the rat brain. Magn Reson Med. 1994;32(4):464-469. doi:10.1002/mrm.1910320407

72. Duong TQ, Kim S-G. In vivo MR measurements of regional arterial and venous blood volume fractions in intact rat brain. Magn Reson Med. 2000;43(3):393-402. doi:10.1002/(SICI)1522-2594(200003)43:3<393::AID-MRM11>3.0.CO;2-K

73. Fournet G, Li J-R, Cerjanic AM, Sutton BP, Ciobanu L, Le Bihan D. A two-pool model to describe the IVIM cerebral perfusion. J Cereb Blood Flow Metab. 2017;37(8):2987-3000. doi:10.1177/0271678X16681310 
74. Wetscherek A, Stieltjes B, Laun FB. Flow-compensated intravoxel incoherent motion diffusion imaging. Magn Reson Med. 2015;74(2):410-419. doi:10.1002/mrm.25410

75. Schneider MJ, Gaass T, Ricke J, Dinkel J, Dietrich O. Assessment of intravoxel incoherent motion MRI with an artificial capillary network: analysis of biexponential and phase - distribution models. Magn Reson Med. 2019;00:1-12. doi: $10.1002 / \mathrm{mrm} .27816$

76. Gardner WJ. Hydrodynamic mechanism of syringomyelia: its relationship to myelocele. J Neurol Neurosurg Psychiatry. 1965;28(3):247.

77. Federau C, Hagmann $P$, Maeder $P$, et al. Dependence of Brain Intravoxel Incoherent Motion Perfusion Parameters on the Cardiac Cycle. Hendrikse J, ed. PLOS ONE. 2013;8(8):e72856. doi:10.1371/journal.pone.0072856

78. Jalnefjord O, Montelius M, Starck G, Ljungberg M. Optimization of b - value schemes for estimation of the diffusion coefficient and the perfusion fraction with segmented intravoxel incoherent motion model fitting. Magn Reson Med. 2019;00:1-12. doi:10.1002/mrm.27826

79. Neil JJ, Bretthorst GL. On the use of bayesian probability theory for analysis of exponential decay date: An example taken from intravoxel incoherent motion experiments. Magn Reson Med. 1993;29(5):642-647. doi:10.1002/mrm.1910290510

80. Ye C, Xu D, Qin Y, et al. Estimation of intravoxel incoherent motion parameters using low b-values. Vegh V, ed. PLOS ONE. 2019;14(2):e0211911. doi:10.1371/journal.pone.0211911

81. While PT. A comparative simulation study of bayesian fitting approaches to intravoxel incoherent motion modeling in diffusion-weighted MRI: Bayesian Fitting Approaches to IVIM Modeling in DWI. Magn Reson Med. 2017;78(6):2373-2387. doi: $10.1002 / \mathrm{mrm} .26598$ 


\title{
Intra-Voxel Incoherent Motion at 7T to quantify human spinal cord perfusion: limitations and promises
}

\author{
Simon Lévy ${ }^{1-4}$, Stanislas Rapacchi ${ }^{1,2}$, Aurélien Massire ${ }^{1,2,4}$, Thomas Troalen ${ }^{5}$, Thorsten \\ Feiweier ${ }^{6}$, Maxime Guye ${ }^{1,2}$, Virginie Callot ${ }^{1,2,4}$
}

\section{Supporting Information}

\author{
Affiliations \\ ${ }^{1}$ Aix-Marseille Univ, CNRS, CRMBM, Marseille, France \\ ${ }^{2}$ APHM, Hopital Universitaire Timone, CEMEREM, Marseille, France \\ ${ }^{3}$ Aix-Marseille Univ, IFSTTAR, LBA, Marseille, France \\ ${ }^{4}$ iLab-Spine International Associated Laboratory, Marseille-Montreal, France-Canada \\ ${ }^{5}$ Siemens Healthcare SAS, Saint-Denis, France \\ ${ }^{6}$ Siemens Healthcare GmbH, Erlangen, Germany
}

\section{Corresponding author}

Virginie Callot

CRMBM-CEMEREM, Aix-Marseille Université, Hôpital Universitaire Timone

27 bd Jean Moulin

13385 Marseille cedex 05, France

Office: +33491388465 | Email: virginie.callot@,univ-amu.fr 


\section{Supporting Information Table S1: Acquisition protocol}

\begin{tabular}{|c|c|c|c|c|c|c|c|c|c|c|c|}
\hline Sequence & Orientation & $\begin{array}{l}\text { Phase-encoding } \\
\text { direction }\end{array}$ & $\begin{array}{l}\text { Resolution } \\
\left(\mathrm{mm}^{3}\right)\end{array}$ & Bandwidth & $\begin{array}{l}\text { FOV/number } \\
\text { of slices }\end{array}$ & $\begin{array}{l}\text { Partial } \\
\text { Fourier }\end{array}$ & iPat & $\begin{array}{l}\text { TE } \\
(\mathrm{ms})\end{array}$ & TR (ms) & $\begin{array}{l}\text { Synchronised } \\
\text { on pulse } \\
\text { oximeter }\end{array}$ & $\begin{array}{l}\text { Purpose and } \\
\text { tips }\end{array}$ \\
\hline $\begin{array}{l}2 \mathrm{D} \\
\text { localizers }\end{array}$ & $\begin{array}{l}\text { Sagittal, } \\
\text { coronal, } \\
\text { axial }\end{array}$ & $2 \mathrm{D}$ & $1.0 \times 1.0 \times 5$ & $260 \mathrm{~Hz} / \mathrm{px}$ & $\begin{array}{l}320 \times 320 \mathrm{~mm}^{2} \text {, } \\
3,7,5 \text { slices } \\
\text { per } \\
\text { orientation }\end{array}$ & $6 / 8$ & None & 3 & 7 & No & $\begin{array}{l}\text { Preliminary } \\
\text { FOV } \\
\text { positioning }\end{array}$ \\
\hline $\begin{array}{l}2 \mathrm{D} \\
\text { saturation- } \\
\text { prepared } \\
\text { turbo-flash }\end{array}$ & Sagittal & $2 \mathrm{D}$ & $1.0 \times 1.0 \times 5$ & 199Hz/px & $\begin{array}{l}264 \times 264 \mathrm{~mm}^{2}, \\
7 \text { slices }\end{array}$ & None & None & 3.05 & 5000 & Yes & $\begin{array}{l}\mathrm{B}_{1} \text { map for coil } \\
\text { voltage } \\
\text { calibration } \\
\text { within } \mathrm{FOV}\end{array}$ \\
\hline $\begin{array}{l}\text { 2D turbo- } \\
\text { spin echo }\end{array}$ & Sagittal & $2 \mathrm{D}$ & $0.6 \times 0.6 \times 2.2$ & $579 \mathrm{~Hz} / \mathrm{px}$ & $\begin{array}{l}192 \times 192 \mathrm{~mm}^{2}, \\
4 \text { slices }\end{array}$ & None & $\begin{array}{l}\text { GRAPPA } \\
2\end{array}$ & 34 & 4814 & Yes & $\begin{array}{l}\text { IVIM FOV } \\
\text { positionning } \\
\text { according to } \\
\text { spinal cord } \\
\text { curvature, } \\
\text { vertebral levels } \\
\text { localization }\end{array}$ \\
\hline $\begin{array}{l}2 \mathrm{D} \mathrm{b}=0 \\
\text { spin-echo } \\
\text { single-shot } \\
\text { EPI }\end{array}$ & Axial & $2 \mathrm{D}$ & $0.77 \times 0.77 \times 5$ & $1130 \mathrm{~Hz} / \mathrm{px}$ & $\begin{array}{l}103 \times 99 \mathrm{~mm}^{2}, \\
6 \text { slices }\end{array}$ & $5 / 8$ & $\begin{array}{l}\text { GRAPPA } \\
3\end{array}$ & 51.6 & $\begin{array}{l}\text { Adapted } \\
\text { to } \\
\text { subject's } \\
\text { cardiac } \\
\text { cycle } \\
\text { and SAR } \\
\text { limits }\end{array}$ & Yes & $\begin{array}{l}\text { Quick check of } \\
\text { shimming and } \\
\text { image quality } \\
\text { (eventual } \\
\text { signal loss, } \\
\text { distortions, } \\
\text { artefacts). } \\
\text { Improve } \\
\text { shimming if } \\
\text { needed. }\end{array}$ \\
\hline
\end{tabular}




\begin{tabular}{|c|c|c|c|c|c|c|c|c|c|c|c|c|}
\hline $\begin{array}{l}\text { 2D multi- } \\
\text { echo } \\
\text { spoiled } \\
\text { gradient- } \\
\text { echo }\end{array}$ & Sagittal & $2 \mathrm{D}$ & & $1.35 \times 1.35 \times 5$ & $981 \mathrm{~Hz} / \mathrm{px}$ & $\begin{array}{l}258 \times 263 \mathrm{~mm}^{2}, \\
13 \text { slices }\end{array}$ & None & None & $\begin{array}{l}4.08 \\
5.1\end{array}$ & 200 & Yes & $\begin{array}{l}\mathrm{B}_{0} \text { map to } \\
\text { inspect } \mathrm{B}_{0} \\
\text { inhomogeneity } \\
\text { within FOV. } \\
\text { Improve } \\
\text { shimming if } \\
\text { too much } \\
\text { variations. }\end{array}$ \\
\hline \multirow{6}{*}{$\begin{array}{l}2 \text { D spin- } \\
\text { echo } \\
\text { single-shot } \\
\text { EPI with } \\
\text { IVIM b- } \\
\text { value } \\
\text { distribution } \\
\text { and } \\
\text { repetitions } \\
\text { number per } \\
\text { b-value as } \\
\text { described } \\
\text { in the } \\
\text { article }\end{array}$} & \multirow[t]{6}{*}{ Axial } & $\begin{array}{l}\text { Diffusion- } \\
\text { encoding }\end{array}$ & $\begin{array}{l}\text { Phase- } \\
\text { encoding }\end{array}$ & \multirow[t]{6}{*}{$0.77 \times 0.77 \times 5$} & \multirow[t]{6}{*}{$1130 \mathrm{~Hz} / \mathrm{px}$} & \multirow[t]{6}{*}{$\begin{array}{l}103 \times 99 \mathrm{~mm}^{2}, \\
6 \text { slices }\end{array}$} & \multirow[t]{6}{*}{$5 / 8$} & \multirow[t]{6}{*}{$\begin{array}{l}\text { GRAPPA } \\
3\end{array}$} & \multirow[t]{6}{*}{51.6} & \multirow{6}{*}{$\begin{array}{l}\text { Adapted } \\
\text { to } \\
\text { subject's } \\
\text { cardiac } \\
\text { cycle } \\
\text { and SAR } \\
\text { limits }\end{array}$} & \multirow[t]{6}{*}{ Yes } & \multirow{6}{*}{$\begin{array}{l}\text { IVIM protocol } \\
\text { split into } 6 \text { runs } \\
\text { ( } 3 \text { diffusion- } \\
\text { encoding } \\
\text { directions with } \\
\text { forward and } \\
\text { reverse phase- } \\
\text { encoding to } \\
\text { correct image } \\
\text { distortions in } \\
\text { post- } \\
\text { processing) }\end{array}$} \\
\hline & & R-L & $\begin{array}{l}\mathrm{R}>\mathrm{L} \\
\mathrm{L}>\mathrm{R}\end{array}$ & & & & & & & & & \\
\hline & & \multirow[t]{2}{*}{$\mathrm{A}-\mathrm{P}$} & $\mathrm{R}>\mathrm{L}$ & & & & & & & & & \\
\hline & & & $\mathrm{L}>\mathrm{R}$ & & & & & & & & & \\
\hline & & \multirow[t]{2}{*}{ I-S } & $\mathrm{R}>\mathrm{L}$ & & & & & & & & & \\
\hline & & & $\mathrm{L}>\mathrm{R}$ & & & & & & & & & \\
\hline $\begin{array}{l}\text { Multi-echo } \\
\text { gradient- } \\
\text { echo }\end{array}$ & Axial & \multicolumn{2}{|l|}{$2 \mathrm{D}$} & $0.4 \times 0.4 \times 5$ & $289 \mathrm{~Hz} / \mathrm{px}$ & $\begin{array}{l}150 \times 150 \mathrm{~mm}^{2}, \\
6 \text { slices }\end{array}$ & $6 / 8$ & $\begin{array}{l}\text { GRAPPA } \\
2\end{array}$ & $\begin{array}{l}3.51 \\
9.34 \\
15.17 \\
21\end{array}$ & 500 & No & $\begin{array}{l}\text { Cord and gray } \\
\text { matter } \\
\text { depiction }\end{array}$ \\
\hline
\end{tabular}

$R-L, A-P, I-S$ : right-left, anterior-posterior and inferior-superior directions. 


\section{Supporting Information Figure S1: Experimental optimization of acquisition parameters}
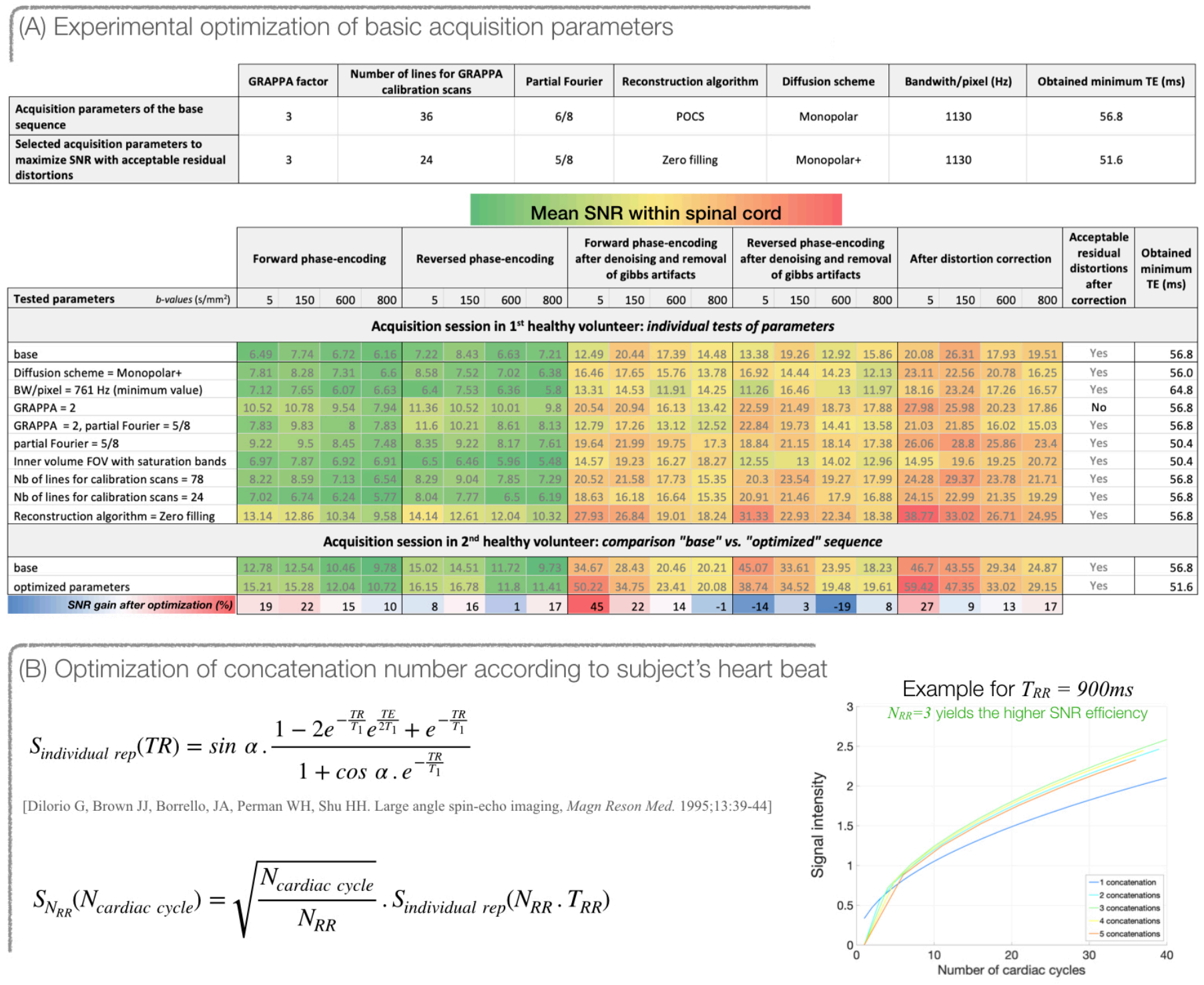
(A) Experimental optimization of basic acquisition parameters. In a first session, parameters were changed one-by-one starting from a parameter set previously defined on phantom ("base" protocol define in top table). SNR was quantified within SC across 8 repetitions of 4 b-values (5, 150 , $600,800 \mathrm{~s} / \mathrm{mm}^{2}$ ) for each parameter set for images acquired with forward and reversed phase-encoding, after denoising and removal of Gibbs artifacts and after distortion correction. Given the low mumber of repetitions, the "difference" method (as described in Reeder et al..$^{58}$ ) was employed here to quantify SNR. Final images were visually inspected in comparison with the anatomic multi-echo gradient-echo image to assess whether distortion correction was satisfactory or whether unacceptable distortions persisted. Based on those results, an "optimized" parameter set was defined to maximize SNR and was evaluated in a second session (different volunteer), across 24 repetitions of the same b-values. The "optimized" parameter set yielded an SNR increase of $27 \%$ compared to the "base" parameter set on $b \simeq 0$ images. (B) Numerical optimization of $N_{R R}$ (number of cycles to acquire all slices, also called number of concatenations) to maximize SNR efficiency. Based on the large angle spinecho signal equation ${ }^{52}\left(\boldsymbol{\alpha}=90^{\circ}, \mathrm{T}_{1}=1251 \mathrm{~ms}\right.$ in $\mathrm{SC}$ at $\left.7 \mathrm{~T}^{22}, \mathrm{TE}=51.6 \mathrm{~ms}\right)$, the optimal $N_{R R}$ can be calculated for the specific cardiac cycle duration of the subject (e.g., for a cardiac cylce of $900 \mathrm{~ms}$, the division of the slices acquisition across 3 cycles yields the higher SNR efficiency). 


\section{Supporting Information Figure S2: Comparison of IVIM fitting approaches for the human}

spinal cord: the two-step segmented approach versus the one-step approach

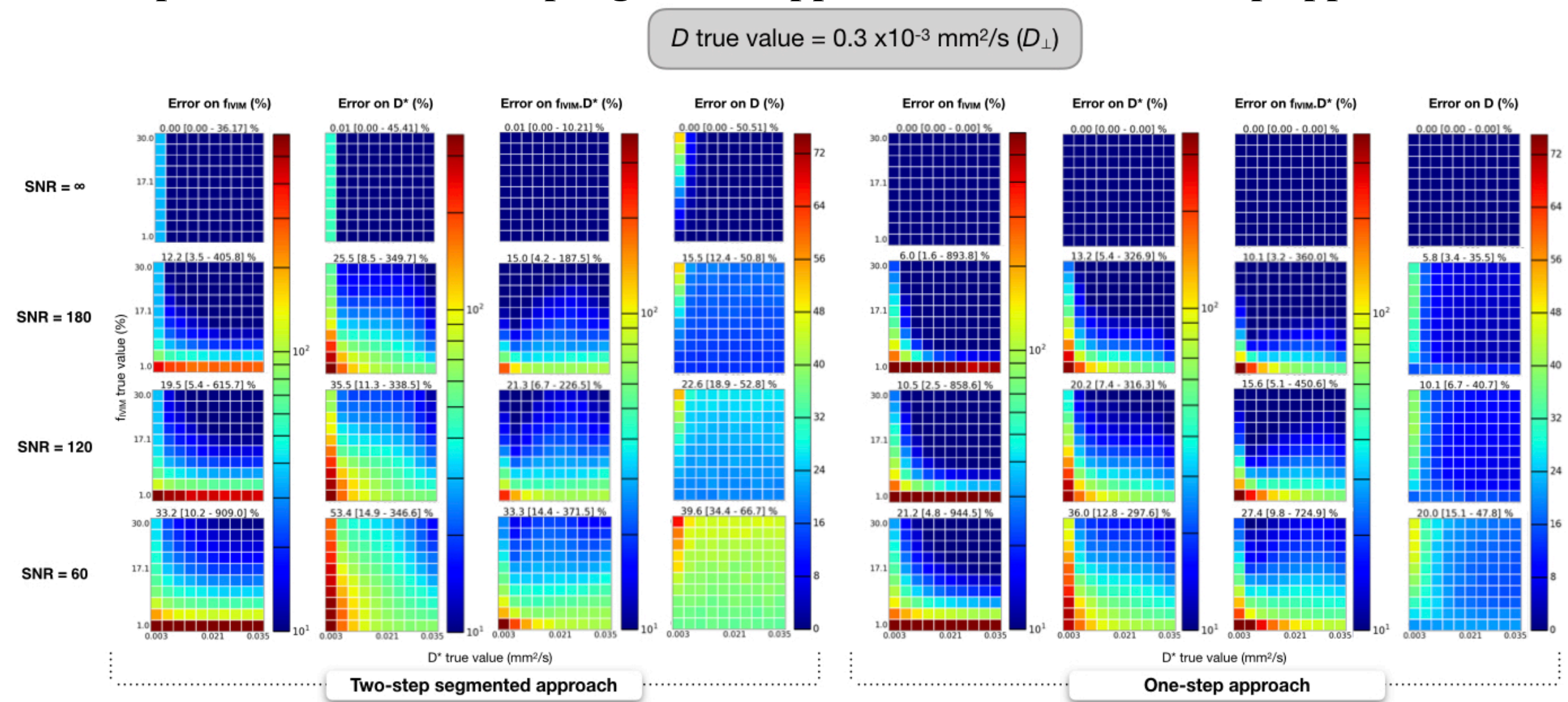

Estimation errors on each IVIM parameter according to $\left(f_{I V I M}, D^{*}\right)$ true values with $D_{\text {true }}=0.3 \times 10^{-3} \mathrm{~mm}^{2} / \mathrm{s}\left(D_{\perp}\right.$, similar to diffusivity in SC transverse plane) for the two fitting approaches and different levels of SNR: $\infty$ (no noise), 180 (maximum measured in-vivo SNR), 120 (mean), 60 (minimum). On the $y$-axis of each graph, $f_{I V I M}$ varies from 1 to $30 \%$ while on the $x$-axis $D^{*}$ varies from 3 to $35 \times 10^{-3} \mathrm{~mm}^{2} / \mathrm{s}$ (bounds defined according to IVIM literature in brain white and gray matter as described in section 2.1). Median [min-max] errors are indicated on top of graphs. 


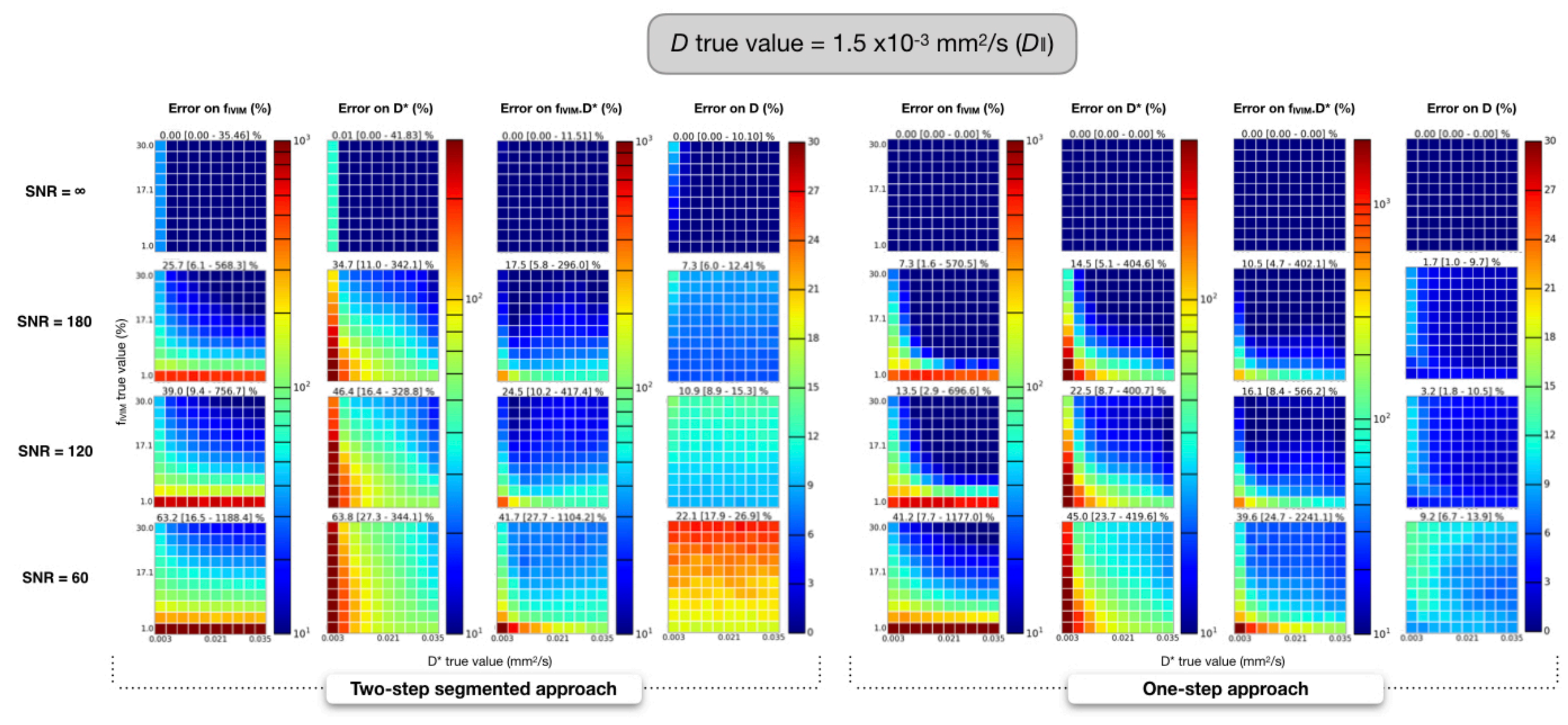

Estimation errors on each IVIM parameter according to $\left(f_{I V I M}, D^{*}\right)$ true values with $D_{\text {true }}=1.5 \times 10^{-3} \mathrm{~mm}^{2} / \mathrm{s}\left(D_{\|}\right.$, similar to diffusivity along SC axis) for the two fitting approaches and different levels of SNR: $\infty$ (no noise), 180 (maximum measured in-vivo SNR), 120 (mean), 60

(minimum). On the $y$-axis of each graph, $f_{I V I M}$ varies from 1 to $30 \%$ while on the $x$-axis $D^{*}$ varies from 3 to $35 \times 10^{-3} \mathrm{~mm}^{2} / \mathrm{s}$ (bounds defined according to IVIM literature in brain white and gray matter as described in section 2.1). Median [min-max] errors are indicated on top of each graph. 


\section{Supporting Information Figure S3: In-vivo SNR distribution within spinal cord for the cohort studied}

Histogram of the in-vivo voxel-wise SNR measured within the entire SC for the whole cohort in lowest $b$-value images $\left(b \leq 35 \mathrm{~s} / \mathrm{mm}^{2}\right.$ ) after the post-processing pipeline was applied. This SNR was calculated voxel-wise as the ratio of the mean signal across repetitions to the SD across repetitions. A factor of $\sqrt{N_{\text {repetitions }}}$ was applied to reflect the SNR value of the average of all repetitions, which is exactly the input data fed into the fitting algorithm. Vertical black lines indicate SNR values used in Monte-Carlo simulations, which are approximately the minimum, maximum and mean values across subjects of the average voxel-wise SNR within SC (60, 180 and 120 respectively).

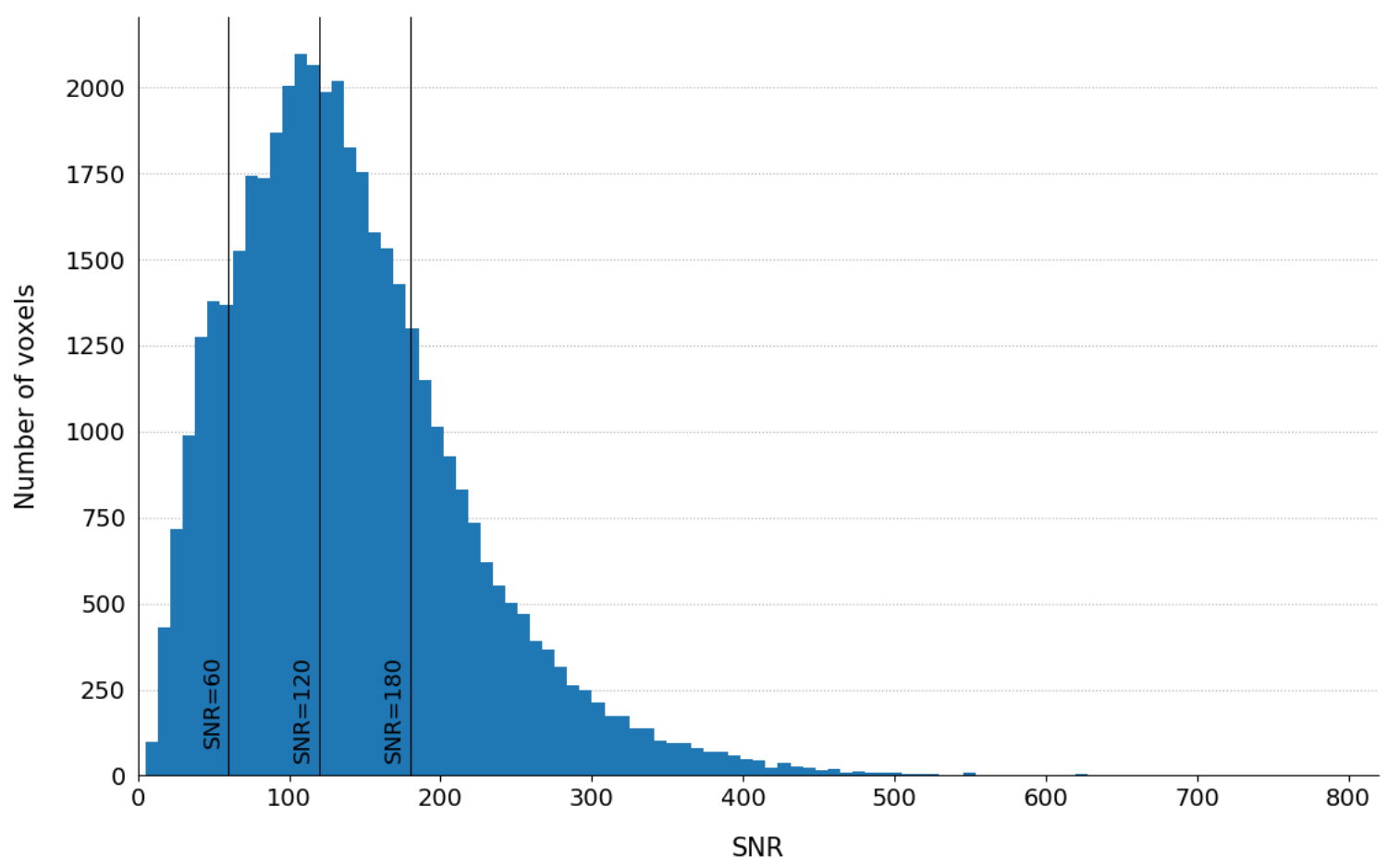

